\title{
A rotational and variability study of a large sample of PMS stars in NGC 2264^
}

\author{
M. H. Lamm ${ }^{1}$, C. A. L. Bailer-Jones ${ }^{1}$, R. Mundt ${ }^{1}$, W. Herbst ${ }^{2}$, and A. Scholz ${ }^{3}$ \\ ${ }^{1}$ Max-Planck-Institut für Astronomie, Königstuhl 17, 69117 Heidelberg, Germany \\ 2 Van Vleck Obs., Wesleyan Univ., Middletown, CT 06459, USA \\ 3 Thüringer Landessternwarte Tautenburg, Sternwarte 5, 07778 Tautenburg, Germany
}

Received 30 June 2003 / Accepted 19 November 2003

\begin{abstract}
We present the results of an extensive search for periodic and irregular variable pre-main sequence (PMS) stars in the young (2-4 Myr) open cluster NGC 2264, based on photometric monitoring using the Wide Field Imager (WFI) on the $2.2 \mathrm{~m}$ telescope on La Silla (Chile). In total, about 10600 stars with $I_{\mathrm{C}}$ magnitudes between 9.8 mag and 21 mag have been monitored in our $34^{\prime} \times 33^{\prime}$ field. Time series data were obtained in the $I_{\mathrm{C}}$ band in 44 nights between Dec. 2000 and March 2001; altogether we obtained 88 data points per star. Using two different time series analysis techniques (Scargle periodogram and CLEAN) we found 543 periodic variable stars with periods between 0.2 days and 15 days. Also, 484 irregular variable stars were identified using a $\chi^{2}$-test. In addition we have carried out nearly simultaneous observations in $V, R_{\mathrm{C}}$ and a narrow-band $\mathrm{H} \alpha$ filter. The photometric data enable us to reject background and foreground stars from our sample of variable stars according to their location in the $I_{\mathrm{C}}$ vs. $\left(R_{\mathrm{C}}-I_{\mathrm{C}}\right)$ colour-magnitude and $\left(R_{\mathrm{C}}-\mathrm{H} \alpha\right)$ vs. $\left(R_{\mathrm{C}}-I_{\mathrm{C}}\right)$ colour-colour diagrams. We identified 405 periodic variable and 184 irregular variable PMS stars as cluster members using these two different tests. In addition 35 PMS stars for which no significant variability were detected could be identified as members using an $\mathrm{H} \alpha$ emission index criterion. This yields a total of 624 PMS stars in NGC 2264, of which only 182 were previously known. Most of the newly found PMS stars are fainter than $I_{\mathrm{C}} \simeq 15 \mathrm{mag}$ and of late spectral type $(\gtrsim \mathrm{M} 2)$. We find that the periodic variables, as a group, have a smaller degree of variability and smaller $\mathrm{H} \alpha$ index than the irregular variables. This suggests that the sample of periodic variables is biased towards weak-line T Tauri stars (WTTSs) while most of the irregular variables are probably classical T Tauri stars (CTTSs). We have quantified this bias and estimated that the expected fraction of WTTSs among PMS stars in the cluster is $77 \%$. This is relatively close to the fraction of WTTSs among the periodic variables which is $85 \%$. We also estimated the total fraction of variables in the cluster using only two well selected concentrations of PMS stars called NGC 2264 N \& S in which we can easily estimate the total number of PMS stars. We find that at least $74 \%$ of the PMS stars in the cluster with $I_{\mathrm{C}} \leq 18.0 \mathrm{mag}$ were found to be variable (either periodic or irregular) by our study. This number shows that our search for PMS stars in NGC 2264 through extensive and accurate photometric monitoring is very efficient in detecting most PMS stars down to at least $I_{\mathrm{C}}=18.0 \mathrm{mag}$.
\end{abstract}

Key words. galaxy: open clusters and associations: individual: NGC 2264 - stars: pre-main sequence - stars: rotation stars: activity - stars: starspots - methods: data analysis

\section{Introduction}

The angular momentum problem is one of the longest standing conundrums in astronomy. Briefly stated, molecular cloud cores have four to six orders of magnitude too much angular momentum to be incorporated into a star. Without shedding their excess angular momentum, stars could not form. When and how they rid themselves of angular momentum is still an open question. It is intimately connected to the issue of disk formation and evolution, to the formation of binary systems and, during the pre-main sequence (PMS) phase, to the

Send offprint requests to: R. Mundt, e-mail: mundt@mpia.de

* Full Tables 4 and 6 are only available in electronic form at the CDS via anonymous ftp to

cdsarc.u-strasbg.fr $(130.79 .128 .5)$ or via

http://cdsweb.u-strasbg.fr/cgi-bin/qcat?]/A+A/417/557 magnetic interaction between stars and disks which likely regulates accretion and drives outflows and jets (e.g. Bodenheimer 1989; Bouvier et al. 1997; Stassun et al. 1999; Mathieu 2003).

Observationally, our best hope of constraining the angular momentum evolution of PMS stars is to obtain stellar rotation rates for objects at a variety of masses and ages. Fortunately, it is possible to do this photometrically because PMS stars have strong surface magnetic fields which produce large cool spots (e.g. Feigelson \& Montmerle 1999). It is often the case that the spot pattern is sufficiently asymmetric and stable that the rotation period can be found by photometric monitoring (e.g. Rydgren \& Vrba 1983; Herbst et al. 1994; Stassun et al. 1999). The advantage of this photometric method compared with $v$ sin $i$ measurements is that it yields directly the rotation period independent of the inclination of the star and it can be very accurate even for slow rotating stars. 
Herbst et al. (2001, 2002) have used this method and obtained rotation periods for $369 \mathrm{~T}$ Tauri stars (TTSs) in the $\sim 1$ Myr old open cluster Orion Nebular cluster (ONC). They found that the period distribution for higher mass stars (i.e. $M \geq 0.25 M_{\odot}$ ) is bimodal with peaks at 2 and 8 days. The bimodality is interpreted as an effect of disk-star interactions in PMS stars: slow rotators have been magnetically locked to their disks which prevent them spinning up with increasing time or equivalently with decreasing radius. Magnetic coupling to an accretion disk was first proposed more than a decade ago as a dominant braking mechanism for PMS stars (Camenzind 1990; Königl 1991; Shu et al. 1994).

However, we also mention a principal limitation of the photometric method for studying the rotational periods among TTSs. As we will show the photometric method can much more easily measure the periods among the weak-line $\mathrm{T}$ Tauri stars (WTTSs) but only for a fraction of the classical T Tauri stars (CTTSs) due to their higher irregular variability. This "photometric noise" often prevents the detection of a periodic signal in the photometric data. Therefore any photometric monitoring of a young open cluster (like the ONC or NGC 2264) will be biased towards WTTSs in the sense that for these stars the fraction of measurable periods will be higher.

From the results of the rotational study in the ONC several questions naturally arise: 1) Is the period distribution similar in other clusters (i.e. does environment play a role)? 2) How many PMS stars interact with their disks and how strongly does this affect the angular momentum evolution? 3) How does the period distribution evolve with time (i.e. do most stars decouple from their disks on time scales of the age of an ONC star and spin up with conserved angular momentum or do disks and disk-locking persist for longer time scales)? To answer these questions it is necessary to measure rotation periods of large samples of PMS stars in clusters with different ages.

Aside from the ONC, the open cluster NGC 2264 is perhaps the best target for a detailed rotational study, since it is sufficiently nearby (760 pc, Sung et al. 1997), fairly populous, and with an estimated age of 2-4 Myr (Park et al. 2000) it is about a factor of 2-4 older than the ONC.

Prior to our study a few monitoring programs have been carried out in NGC 2264 (e.g. Kearns \& Herbst 1998) which altogether yielded only about 30 published rotation periods for PMS stars. Vogel \& Kuhi 1981 and Soderblom et al. (1999) reported rotational velocities $(v \sin i)$ for in total about 60 low mass NGC 2264 stars. An early variability study has been carried out by Nandy \& Pratt (1972) who identified 26 optical variable stars in the cluster. Neri et al. (1993) reported optical and near-infrared photometry for 50 stars in NGC 2264 and detected for $70 \%$ of these stars optical variability. Due to the limiting sensitivity of these studies most of the stars have spectral types earlier than K5.

About 200 PMS stars and some 20 PMS candidates have been identified in NGC 2264 prior to our study using a variety of methods including $\mathrm{H} \alpha$ spectroscopy (Herbig 1954; Ogura 1984; Marcy 1980; Rydgren 1979), H $\alpha$ narrow band photometry (Sung et al. 1997; Park et al. 2000) or ROSAT X-ray flux measurements (e.g. Flaccomio et al. 2000). A
PMS membership catalogue that summarises (most) these results is available in Park et al. (2000).

Vasilevskis et al. (1965) have carried out a proper motion study of 245 stars in the region of the cluster. However, the suggested membership probabilities must be handled with caution because several stars with strong $\mathrm{H} \alpha$-emission which clearly must be members have low membership probability while other (non-emission) stars with high membership probability are clearly no members (see e.g. the $\mathrm{H} \alpha$-emission study by Rydgren 1979). King (1998) and Soderblom et al. (1999) have in total determined lithium abundances of 35 cluster members. Recently, circumstellar disk candidates have been identified in NGC 2264 by Rebull et al. (2002).

In this paper we present the results of an extensive photometric monitoring program in NGC 2264, in which we monitored $\sim 10600$ stars over a very broad magnitude range $(9.8 \leq$ $\left.I_{\mathrm{C}} \leq 21\right)$. This extensive monitoring program allowed us to discover about 400 new PMS stars in the cluster and in addition we could measure the rotation periods of 405 PMS stars down to the substellar limit.

In the following section we describe the observational and data reduction strategy. Section 3 describes the methods we have used for obtaining absolute and relative photometry. In Sect. 4 we describe the time series analysis. In Sect. 5 we discuss in detail how we selected the PMS stars among the periodic and irregular variables we have found. The nature of the stellar variability is examined in Sect. 6. The identification of additional PMS members for which no variability could be detected is described in Sect. 7. In Sect. 8 we estimate the fraction of variable PMS stars and the completeness level of our PMS sample. In Sect. 9 we investigate if our sample of periodic variables is representative for all cluster members. Our major conclusions are summarised in Sect. 10. The obtained extensive rotational period data and possible consequences for the disk-locking scenario will be discussed in a separate paper (see Lamm et al. 2003, hereafter Paper II).

\section{Data acquisition and reduction}

The photometric monitoring program described here was carried out in the $I_{\mathrm{C}}$ band on 44 nights during a period of two month between 30 Dec. 2000 and 01 Mar. 2001 with the Wide Field Imager (WFI) on the MPG/ESO $2.2 \mathrm{~m}$ telescope on La Silla (Chile). The WFI consists of a mosaic of four by two CCDs with a total array size of $8 \mathrm{k} \times 8 \mathrm{kk}$. The field of view is $34^{\prime} \times 33^{\prime}$ and the scale is $0.238^{\prime \prime} /$ pixel. In Fig. 1 we show a $500 \mathrm{~s} R_{\mathrm{C}}$ exposure of our observed field from which the positions of the eight CCDs is evident. To avoid highly saturated images and light scattering from the very bright star S Mon ( $V=4.7 \mathrm{mag}$ ) this star was located near the northern end of the $23^{\prime \prime} .3$ (96 pixels) wide central gap between two chips. The central position on the sky was close to $\mathrm{RA}(2000)=6^{\mathrm{h}} 40^{\mathrm{m}} 59^{\mathrm{s}}$ and $\operatorname{Dec}(2000)=9^{\circ} 38^{\prime} 59^{\prime \prime}$ for most frames. The typical deviation from this nominal position is $2^{\prime \prime}$. About $5 \%$ of the imaged area is lost due to the gaps between the chips and the small dithering of the frames. In order to increase the dynamical range of the observations, three consecutive exposures of $5 \mathrm{~s}$, $50 \mathrm{~s}$ and $500 \mathrm{~s}$ were taken with the $I_{\mathrm{C}}$ filter. This series of three 

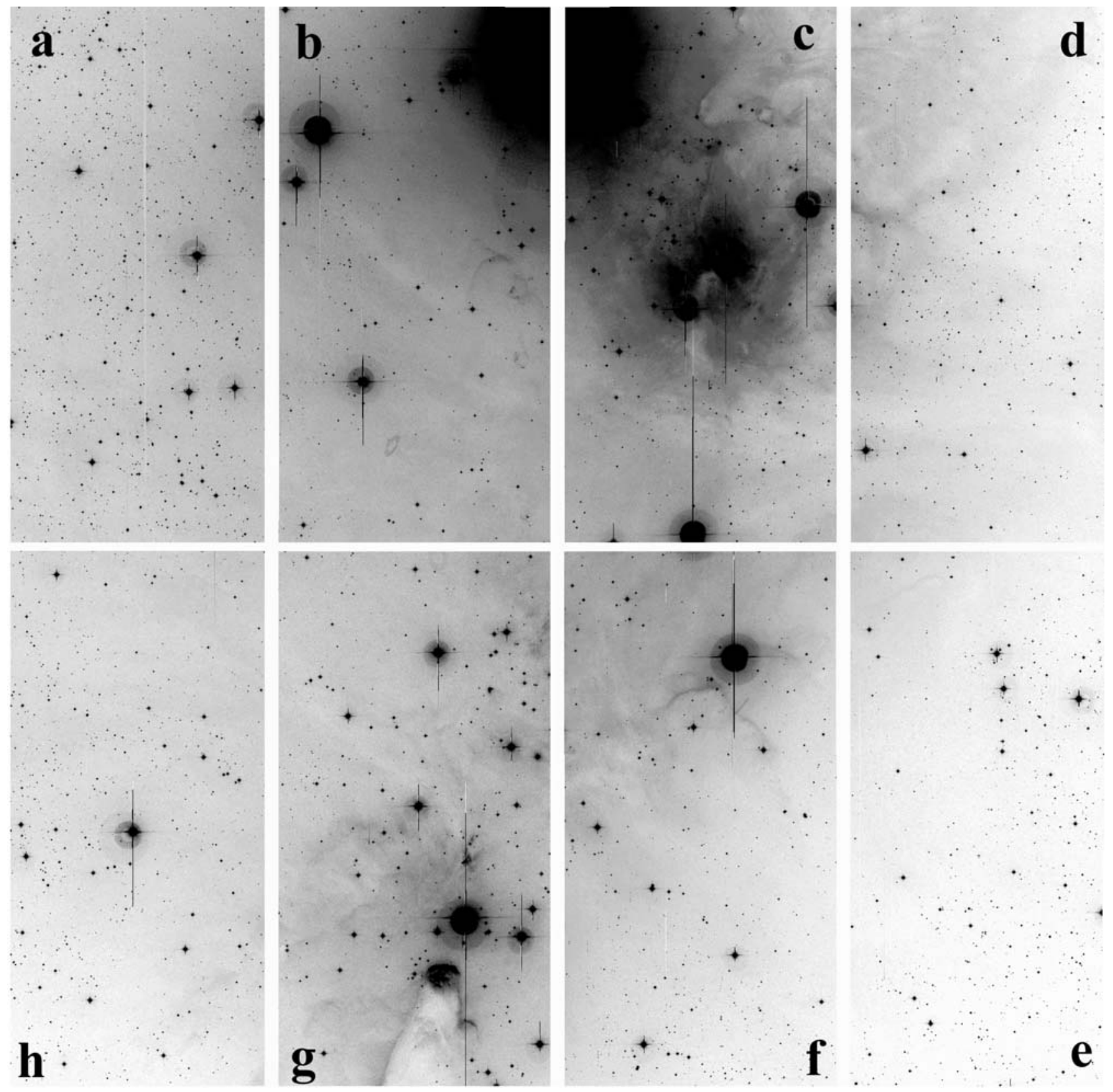

Fig. 1. Reproduction of a $500 \mathrm{~s} R_{\mathrm{C}}$ band exposure of our observed field. The area imaged by the individual chips a-h is quite evident. The bright star near the northern end of the gap between chip b and c is S Mon $(V=4.7)$. In the second chip g the famous cone nebular is visible, which is hardly evident on our $I_{\mathrm{C}}$ band images as is the case for the nebulosity in the centre of chip e.

exposures is defined here as one data point. In total we obtained 110 data points and between 1 and 18 data points per night. The observing time distribution of our time series is shown in Fig. 2. The typical seeing (measured by the FWHM of the PSF) in these images was of the order of $0{ }^{\prime} 8-1{ }^{\prime \prime} 2$.

In addition to the $I_{\mathrm{C}}$ observations we observed the cluster through $V$ and $R_{\mathrm{C}}$ filters on six nights during the 2000/2001 season. In $R_{\mathrm{C}}$ the exposure times were $5 \mathrm{~s}, 50 \mathrm{~s}$ and $500 \mathrm{~s}$, while the exposure times through the $V$ filter were set to $5 \mathrm{~s}$, $60 \mathrm{~s}$ and $720 \mathrm{~s} . I_{\mathrm{C}}$ observations were obtained directly before or after an observation in $V$ or $R_{\mathrm{C}}$. This allows us to determine the $\left(V-I_{\mathrm{C}}\right)$ and $\left(R_{\mathrm{C}}-I_{\mathrm{C}}\right)$ colours from nearly simultaneous measurements in the two filters, i.e. the colours should not be affected by variability to any substantial extent.

In order to improve the absolute photometry, additional $V$, $R_{\mathrm{C}}$ and $I_{\mathrm{C}}$ WFI data were obtained between October 2001 and March 2002 using the same exposure times. In order to search for $\mathrm{H} \alpha$ emission, three consecutive $\mathrm{H} \alpha$ observations of $150 \mathrm{~s}$, $1200 \mathrm{~s}$ and $1200 \mathrm{~s}$ exposure time were taken directly before or after the observations in $R_{\mathrm{C}}$ in order to obtain nearly simultaneously observations in these two filters for a determination of the $\left(R_{\mathrm{C}}-\mathrm{H} \alpha\right)$ colour. The used $\mathrm{H} \alpha$ filter has a central wavelength $\lambda=6588 \AA$ and $F W H M=74 \AA$. A detailed list of observations in filters other than $I_{\mathrm{C}}$ is given in Table 1 . 




Fig. 2. Distribution of observation times of the $I_{\mathrm{C}}$ band time series (between 30 Dec. 2000 and 01 Mar. 2001). The time (expressed as a fraction of a day) as a function of the modified Julian day (MJD) is shown.

Table 1. Dates of observations with the $V, R_{\mathrm{C}}$ and $\mathrm{H} \alpha$ filter for the two observing seasons 2000-2001 and 2001-2002. Listed are the number of data points (i.e. three images of three different exposure times) for each night in a given filter. The observations in $V$ and $R_{\mathrm{C}}$ were take directly before or after a set of three images in $I_{\mathrm{C}}$.

\begin{tabular}{cccc}
\hline \hline Date of & \multicolumn{3}{c}{ data points in } \\
observation & $V$ & $R_{\mathrm{C}}$ & $\mathrm{H} \alpha$ \\
\hline 9 Jan. 2001 & 1 & - & - \\
10 Jan. 2001 & 1 & - & - \\
11 Jan. 2001 & 1 & - & - \\
12 Jan. 2001 & 1 & 1 & - \\
15 Jan. 2001 & - & 1 & - \\
16 Jan. 2001 & - & 1 & - \\
\hline 25 Nov. 2001 & 1 & 1 & 1 \\
11 Dec. 2001 & 1 & - & - \\
4 Jan. 2002 & 1 & 1 & 1 \\
13 Jan. 2002 & 1 & - & - \\
17 Jan. 2002 & 1 & - & - \\
2 Mar. 2002 & 1 & 1 & 1 \\
\hline Total & 10 & 6 & 3 \\
\hline & & &
\end{tabular}

The image processing was done for each of the eight WFI chips separately using the standard IRAF tasks. A onedimensional bias was subtracted from each frame using the overscan region in each frame. A small two-dimensional residual remained which was removed using zero integration time (bias) frames. For images through $V, R_{\mathrm{C}}$ and $\mathrm{H} \alpha$ filters the variable sensitivity across the chips was corrected using the median combination of typically $10-15$ twilight flats taken in the beginning and the end of two or three different nights. In $I_{\mathrm{C}}$ we
Table 2. Number of detected sources in the various chips of the reference images.

\begin{tabular}{c|rrrrrrrrr}
\hline \hline $\begin{array}{c}\text { Exposure } \\
\text { time }\end{array}$ & $\mathrm{a}$ & $\mathrm{b}$ & $\mathrm{c}$ & $\mathrm{d}$ & $\mathrm{e}$ & $\mathrm{f}$ & $\mathrm{g}$ & $\mathrm{h}$ & total \\
\hline $5 \mathrm{~s}$ & 505 & 268 & 445 & 311 & 405 & 278 & 286 & 334 & 2832 \\
$50 \mathrm{~s}$ & 864 & 447 & 586 & 883 & 814 & 546 & 432 & 648 & 5220 \\
$500 \mathrm{~s}$ & 2220 & 900 & 1187 & 1535 & 1754 & 1054 & 654 & 1645 & 10940 \\
\hline
\end{tabular}

used illumination-corrected dome flats for flatfielding the science images. This was necessary because the $I_{\mathrm{C}}$ twilight flats showed interference fringes caused by narrow band emission from the Earth's atmosphere. The illumination-corrected flats were created in the way described by Bailer-Jones \& Mundt (2001). Since the science images in the $I_{\mathrm{C}}$-band were affected by fringing too, it was essential that these fringes be removed. We note that fringing is an additive effect and therefore the fringe pattern has to be subtracted from the science images. Since the fringe pattern was found to be stable over a given observation run we created only one fringe image for each chip per observing season (for more details see Bailer-Jones \& Mundt 2001).

\section{Photometry and astrometry}

Our goal was to perform relative and absolute photometry for every star in our field with a sufficient signal-to-noise ratio, i.e. $S / N=10$ or better. The relative and absolute photometry is described in the following subsections separately. As a common first step we had to identify the positions of all sources in our field. Therefore we first created a source list for all 8 chips employing the DAOFIND task, with a separate list for the $5 \mathrm{~s}$, $50 \mathrm{~s}$ and $500 \mathrm{~s}$ exposures. For this source search we selected for each of the three $5 \mathrm{~s}, 50 \mathrm{~s}$ and $500 \mathrm{~s}$ exposures one frame with a position very close to the nominal central location of all frames. These images we designate here as reference frames. The number of sources detected in each chip of these reference images is listed in Table 2. The coordinates of each source in all other frames were calculated by applying a derived linear offset between the reference images and these frames. The offsets were calculated for each chip separately by measuring the pixel position of a bright star close to the chip's centre in all frames. For the whole observed field the offsets were calculated by using eight bright stars. During the aperture photometry all sources were re-centered (see below) and therefore the offsets calculated in that way are sufficient for identifying the stars in the all other frames.

The IRAF tasks CCXYMATCH and CCMAP were used to cross-identify our sources in the reference frames with objects in the USNO-A2.0 catalogue (Monet et al. 1998) and to calculate the plate transformations to the sky positions. We typically used 70 reference stars (minimum 30, maximum 133) for the calculation of the plate solutions in each chip. Using these plate solutions the final J2000 coordinates of our sources were calculated with the task CCTRAN. These coordinates are listed in Table 4. The rms of the deviations between the fit and the coordinates of the reference stars is typically $0 . ' 1$. 


\subsection{Relative photometry}

All the variability studies described here are based on differential photometry relative to a set of non-variable reference stars. Before the final analysis and after various tests, we rejected some frames from the further analysis. These were usually frames that were taken under poor transparency conditions, poor seeing or with a bright background due to scattered moon light. In total we rejected 22 of the 110 data points before the final analysis, so that relative photometry was performed on 88 remaining data points.

The DAOPHOT/APPHOT task was used to measure the brightness of each object in the source lists. The aperture was chosen to be 8 pixels (1'.9) in diameter for all measurements in order to maximise the signal-to-noise ratio. The sky was calculated for each source separately as the median of an annulus with an inner diameter of 30 pixel $\left(77^{\prime \prime} 1\right)$ and a width of 8 pixel centred on the source. For the calculation of the sky value we used a sigma clipping rejection criterion with a $3 \sigma$-threshold. During the measurement the sources were recentred. Sources with any pixel entering the $\leq 1 \%$ nonlinearity region were rejected.

The differential magnitudes for all sources were formed as follows. First a set of non-variable comparison stars was selected from all sources in each chip for each of the three different exposures. Comparison stars were chosen according to the following criteria: 1) present on every of the 88 frames; 2) isolated from other sources and the corners of the field (see below); 3) the photometric error given by APPHOT (i.e. Poisson error in the source and scatter in the background) is on average less than 0.01 mag. Only stars that passed all of these three tests were selected. Possible variables among the candidate comparison stars were identified by comparing the flux of each candidate with the mean flux of all other candidates at the different epochs. Stars that show the largest variability were rejected from the candidate sample and the procedure was performed again with the remaining stars. After a few iterations typically ten non-variable comparison stars were identified in each chip so that the mean standard deviation in the relative light curves of the comparisons stars (relative to the other comparison stars) was typically $\sigma=0.009$ mag. In the best cases we achieved a mean standard deviation of $\sigma=0.006$ mag which is therefore the maximum precision we can expect for the relative photometry. This limit is most likely set by flat fielding errors and not by photon noise.

The selected comparison stars were used for the calculation of the relative magnitude $m_{\text {rel }}\left(t_{i}\right)$ of each source in the field at the different epochs $t_{i}$. The relative magnitude and its error $\delta m_{\text {rel }}\left(t_{i}\right)$ were calculated as described in Bailer-Jones \& Mundt (2001). We note that the mean was subtracted from each light curve so that $\sum_{i} m_{\text {rel }}\left(t_{i}\right)=0$.

In Fig. 3 we show the mean error $\overline{\delta m_{\text {rel }}}$ in a single measurement as a function of magnitude for the stars measured with the three different exposure times (for the magnitude determination see Sect. 3.2). As an example we show the light curves of four stars in Fig. 4.
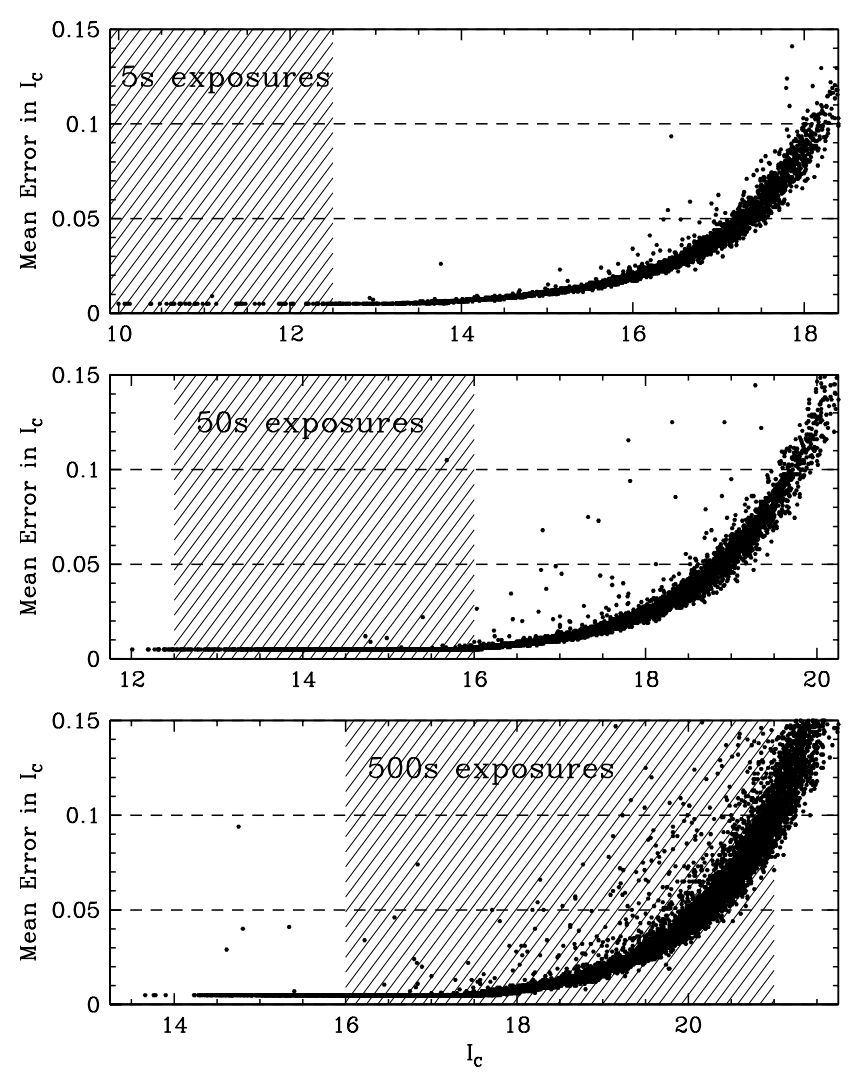

Fig. 3. The mean error $\overline{\delta m_{\text {rel }}}$ in a single measurement for the three different exposure times of $5 \mathrm{~s}$ (top), $50 \mathrm{~s}$ ( mid) and $500 \mathrm{~s}$ (bottom) as a function of $I_{\mathrm{C}}$ magnitude. The shaded regions indicate which exposure time we used for the further analysis of a star with a given magnitude (see text). Note the different magnitude scales in the three plots.

\subsection{Absolute photometry}

In order to obtain additional constraints on the nature of the observed stars, in particular on their PMS membership, we obtained absolute photometry in $V, R_{\mathrm{C}}$ and $I_{\mathrm{C}}$. As we will outline below all of our photometry is done relative to secondary standards in our observed field. The result of the absolute photometry is reported in Table 4.

Many of the stars in the field are expected (and found, see Sect. 4) to be variable and the peak-to-peak variations of the variable stars are in most cases $\$ 0.2 \mathrm{mag}$ in $I_{\mathrm{C}}$ (see Fig. 13). Therefore a single measurement of the magnitude of a star depends on the phase and amplitude of the star's brightness modulation. On the other hand single measurements of the colour of a PMS star with cool (or hot) surface spots also differ at different epochs. That is because the spectral energy distribution of the light coming from the area of the spot differs from the spectral energy distribution of the light radiated from the other part of the stellar atmosphere because of the different effective temperatures. The difference between the two energy distributions is wavelength dependent and larger for shorter wavelengths. Therefore, if cool spots cause the variability of a star the peakto-peak variation in its light curve increases to shorter wavelength, i.e. it is larger in the $V$-band and smaller in the $I_{\mathrm{C}}$ band. Observations of T Tauri stars confirm this expected colour behaviour of the stars (e.g. Vrba et al. 1989; Herbst et al. 1994). 

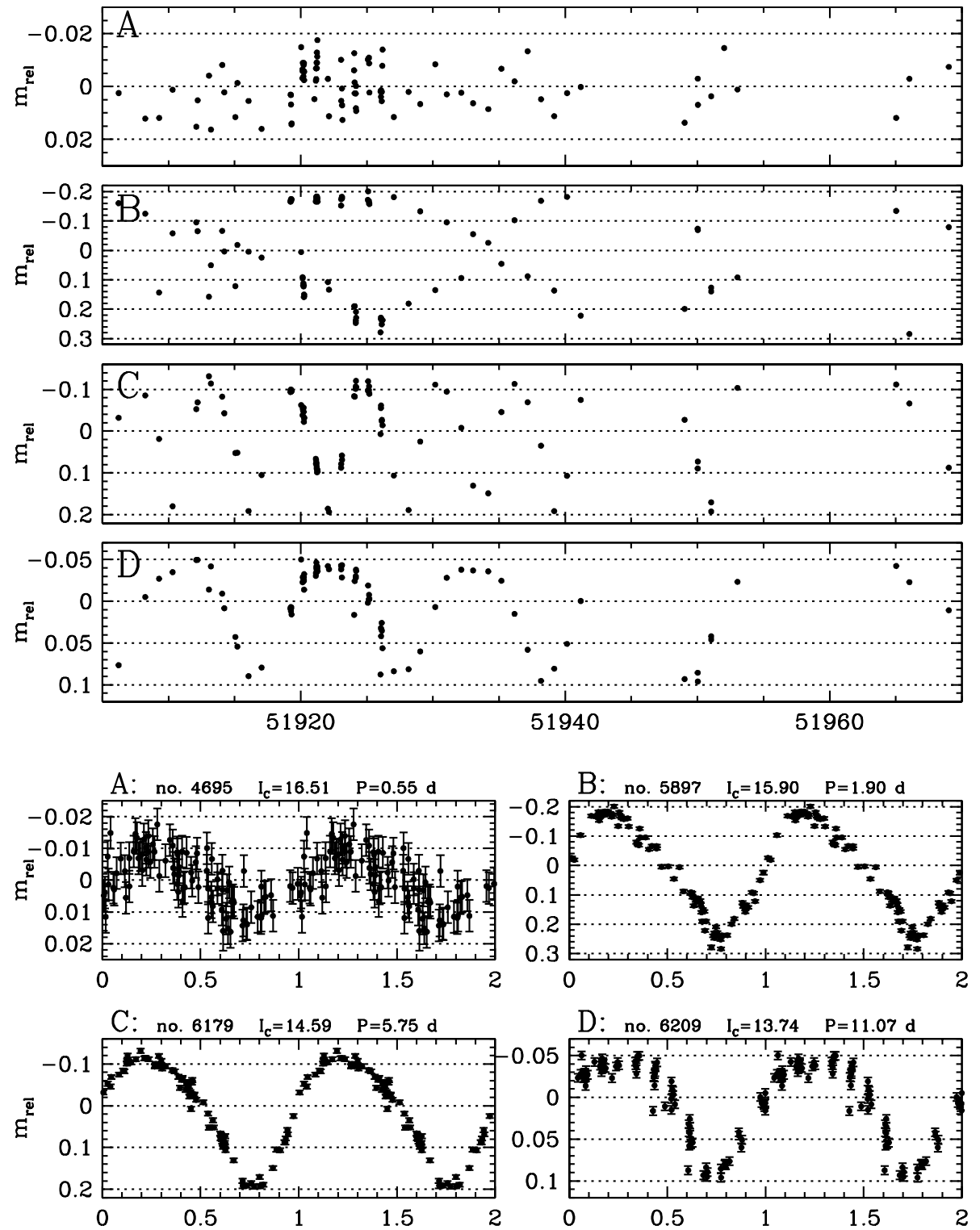

Fig. 4. Examples for relative light curves (mean subtracted) of four stars and the resulting phased light curve for each of the four stars. The light curves were phased with the period that we found by the Scargle periodogram analysis technique. For each star we also list the Number (No.), the mean $I_{\mathrm{C}}$ magnitude and the period $P$.

The relations between the peak-to-peak variations $\Delta V, \Delta R_{\mathrm{C}}$ and $\Delta I_{\mathrm{C}}$ in the different filters are given by the colour slopes

$S_{R}=\frac{\mathrm{d}\left(\Delta R_{\mathrm{C}}-\Delta I_{\mathrm{C}}\right)}{\mathrm{d}\left(\Delta I_{\mathrm{C}}\right)} \quad$ and $\quad S_{V}=\frac{\mathrm{d}\left(\Delta V_{\mathrm{C}}-\Delta I_{\mathrm{C}}\right)}{\mathrm{d}\left(\Delta I_{\mathrm{C}}\right)}$.

Herbst et al. (1994) calculated these slopes for several WTTSs with a mean value of $S_{R}=0.31 \pm 0.23$ and $S_{V}=0.55 \pm 0.36$. Since the typical peak-to-peak variations we found in our target stars are of the order of $\Delta I_{\mathrm{C}} \simeq 0.2$ we expect maximal colour changes of about $\Delta\left(R_{\mathrm{C}}-I_{\mathrm{C}}\right) \simeq 0.06$ and $\Delta\left(V-I_{\mathrm{C}}\right) \simeq 0.1$ but in some extreme cases the variations in the colour may be higher.

To take into account these magnitude and colour changes we determined the $I_{\mathrm{C}}$ magnitude and the $\left(V-I_{\mathrm{C}}\right)$ and $\left(R_{\mathrm{C}}-I_{\mathrm{C}}\right)$ colours at different epochs. The colours and magnitudes we list in Table 4 are average values of these different measurements. The dates of the employed measurements are listed in Table 1.
For the determination of the average $I_{\mathrm{C}}$ magnitude of each star we used only images which were taken during the first observing season in Jan. 2001. In this season we got nearly simultaneous measurements in $I_{\mathrm{C}}$ and $R_{\mathrm{C}}$ (i.e. three images in $I_{\mathrm{C}}$ with different exposure times followed by three images of different exposure times in $R_{\mathrm{C}}$ ) or nearly simultaneous measurements in $I_{\mathrm{C}}$ and $V$ at seven different epochs (see Table 1). The final averaged $\left(R_{\mathrm{C}}-I_{\mathrm{C}}\right)$ colours were calculated from nearly simultaneous measurements in $R_{\mathrm{C}}$ and $I_{\mathrm{C}}$ at the six different epochs during both observing seasons listed Table 1 . The averaged $\left(V-I_{\mathrm{C}}\right)$ colours were calculated from nearly simultaneous measurements in $V$ and $I_{\mathrm{C}}$ at the 10 different epochs in both observing seasons listed in Table 1. The transformation of our instrumental $V_{\text {instr }}, R_{\mathrm{C} \text {,instr }}$ and $I_{\mathrm{C} \text {,instr }}$ magnitudes into the true $\left(V-I_{\mathrm{C}}\right)$ and $\left(R_{\mathrm{C}}-I_{\mathrm{C}}\right)$ colours and $I_{\mathrm{C}}$ magnitude is outlined below. It was done before the averaging and for each 
epoch, each chip and each exposure time separately using a linear transformation.

Since we did not observe any flux standards during any of the observing seasons we had to use secondary standard stars located in our field for calibrating our measurements. The photometric calibration coefficients were determined using magnitudes and colours of stars in NGC 2264 measured by Rebull et al. (private communication). Their photometric data include the photometry of PMS stars in the cluster from Rebull et al. (2002) and in addition unpublished photometry of foreground and background objects in their observed field. This extended dataset including the unpublished data, compared to a dataset consisting only of PMS stars, has the advantage of a smaller fraction of variable stars. Our objects were cross-identified with the 2924 objects in the extended Rebull et al. catalogue that are located in our field. The 2227 (76\%) stars which we could identify we used as secondary photometric standard stars.

We transformed our instrumental magnitudes $I_{\mathrm{C} \text {,instr }}$ into the Cousins $I$ system by applying the linear transformation equations of the form

$I_{\mathrm{C}}=a_{1}+b_{1} \times\left(R_{\mathrm{C}, \text { instr }}-I_{\mathrm{C}, \text { instr }}\right)+I_{\mathrm{C}, \text { instr }} \quad$ and

$I_{\mathrm{C}}=a_{2}+b_{2} \times\left(V_{\mathrm{instr}}-I_{\mathrm{C}, \text { instr }}\right)+I_{\mathrm{C}, \text { instr }}$

to the measurements in $I_{\mathrm{C}}$ which were nearly simultaneous with one of the other two filters $V$ or $R_{\mathrm{C}}$. The coefficients $a_{1}$ and $b_{1}$ were calculated simultaneously using the secondary photometric standard stars by applying a linear least squares fit to the points in the $\left(I_{\mathrm{C}, \text { Rebull }}-I_{\mathrm{C} \text {,instr }}\right)$ vs. $\left(R_{\mathrm{C} \text {,instr }}-I_{\mathrm{C} \text {,instr }}\right)$ plane, where $I_{\mathrm{C} \text {,Rebull }}$ is the magnitude in our secondary standard catalogue. The coefficients $a_{2}$ and $b_{2}$ were calculated in the same way using the $\left(I_{\mathrm{C}, \text { Rebull }}-I_{\mathrm{C} \text {,instr }}\right)$ vs. $\left(V_{\text {instr }}-I_{\mathrm{C} \text {,instr }}\right)$ plane.

We typically used between 100 and 200 stars per chip and exposure time (minimum 42, maximum 392) for calculating the linear transformation coefficients. Thus even though many of the standard stars are variable this large number ensures a robust transformation. Obvious outliers were rejected before the calculation of the coefficients was done. Since the variable stars were in different phases of their brightness modulation for a given epoch the related data points scatter around the true transformation function. The uncertainties of the transformation coefficients results from this scatter. The median values of the slopes are $b_{1}=0.18$ and $b_{2}=0.10$. The typical uncertainties of the transformation coefficients are $\delta a_{1}=0.005$ and $\delta b_{1}=0.010$ for the first and $\delta a_{2}=0.015$ and $\delta b_{2}=0.010$ for the second equation. With this method we derived values for $I_{\mathrm{C}}$ at 7 different epochs between 9 Jan. 2001 and 16 Jan. 2001.

The final $I_{\mathrm{C}}$ magnitude for each star was calculated as the average of these 7 different $I_{\mathrm{C}}$ measurements. To improve the results we make use of the fact that we know the phase and amplitude of each star at these epochs from the relative photometric analysis described in Sect. 3.1. Before the final averaging we subtracted the relative magnitude $\left(m_{\mathrm{rel}}\right)$ of the corresponding data point in the (mean subtracted) relative light curve from the calculated $I_{\mathrm{C}}$ magnitude at each epoch. The mean of the resulting values is listed in Table 4 .

The errors for this final $I_{\mathrm{C}}$ magnitudes were estimated in two different ways. First, we calculated the expected $1 \sigma$ error $\delta I_{\mathrm{C} \text {,exp }}$ from the measured IRAF errors ${ }^{1}$ and the errors of the transformation coefficients. Second, we calculated the standard deviation $\sigma_{7}$ of the 7 independent $I_{C}$ measurements. The final assigned error to the magnitude is the maximum of this two error estimations for each star: $\delta I_{\mathrm{C}}=\max \left(\delta I_{\mathrm{C}, \exp }, \sigma_{7}\right)$.

The colours in the Johnson $V$ and Cousins $R, I$ system were calculated in a similar way. The transformation from instrumental colour to the true colour was done using the transformation equations

$$
\begin{aligned}
& \left(R_{\mathrm{C}}-I_{\mathrm{C}}\right)=c_{1}+d_{1} \times\left(R_{\mathrm{C}, \text { instr }}-I_{\mathrm{C}, \text { instr }}\right) \quad \text { or } \\
& \left(V-I_{\mathrm{C}}\right)=c_{1}+d_{1} \times\left(V_{\mathrm{instr}}-I_{\mathrm{C}, \text { instr }}\right) .
\end{aligned}
$$

Again, the transformation coefficients were calculated using a least square linear fit. The median values of the slopes are $d_{1}=$ -0.26 and $d_{2}=-0.17$. With this method we derived ten values for $\left(V-I_{\mathrm{C}}\right)$ and six for $\left(R_{\mathrm{C}}-I_{\mathrm{C}}\right)$. The final colours for each star were obtained by calculating the mean of these values for each colour. The errors were derived in the same way as described above.

\subsection{Final photometry database}

The absolute photometric calibration described in the previous section was done for each chip and exposure time separately. The objects that were measured on the same chip with two different exposure times were identified in order make sure that they do not appear twice in the final catalogue. For stars with $I_{\mathrm{C}} \geq 16.0 \mathrm{mag}$ the colours and magnitudes listed in Table 4 were taken from the $500 \mathrm{~s}$ exposures. The measurements for stars with $12.5 \mathrm{mag} \leq I_{\mathrm{C}} \leq 16.0$ and with $10 \mathrm{mag} \leq I_{\mathrm{C}} \leq 12.5 \mathrm{mag}$ were taken from the $50 \mathrm{~s}$ and $5 \mathrm{~s}$ exposures, respectively. Thus we list the measurements with the highest signal-to-noise for each star and avoid pixel saturation in images with the best seeing. The magnitude ranges used for our data analysis of the three different exposure times are also indicted in Fig. 3.

We compared the results of our photometry with those of other authors. In Table 3 we report the mean offsets in the photometry between our study and those of other studies. We did not find any significant difference between our results and those reported by Rebull et al. (2002), Park et al. (2000), and Sung et al. (1997). The small differences between the studies can be explained by intrinsic stellar variability. However, there is a significant offset in the photometry compared the the study of Flaccomio et al. (2000). The reason therefor could be different transmission curves of the used filters and a different average colour of the employed flux standards.

A small part of the final photometric catalogue is shown in Table 4 and the complete table that contains all 10554 stars is available electronically. In Table 4 we also list the $\left(R_{\mathrm{C}}-\mathrm{H} \alpha\right)$ colour of the stars. Since no $\mathrm{H} \alpha$ standard star measurements were available only the instrumental colour is given. The limiting magnitudes in the different pass-bands for different signal-to-noise levels is given in Table 5.

\footnotetext{
1 The IRAF errors were corrected to somewhat higher values as described in Sect. 4.2.
} 
Table 3. Comparison of our photometry with those of other authors. We list the offsets in the sense results of other author minus our results. We also show the number of stars which we used for the comparison with other studies. These numbers differ for a single publication because not all magnitudes or colours were available for all stars. Stars with close-by neighbours were not not used for the calculation of the offsets, since these stars are not separated in the other studies.

\begin{tabular}{lcc|cc|cc}
\hline \hline \multicolumn{1}{c}{ Publication } & No. of stars & $I_{\mathrm{C}}$ & No. of stars & $\left(R_{\mathrm{C}}-I_{\mathrm{C}}\right)$ & No. of stars & $\left(V-I_{\mathrm{C}}\right)$ \\
\hline Rebull et al. (2002) & 1344 & $0.003 \pm 0.002$ & 1047 & $-0.019 \pm 0.002$ & 767 & $-0.010 \pm 0.002$ \\
Park et al. (2000) & 147 & $-0.009 \pm 0.012$ & $\ldots \ldots \ldots \ldots$ & $\ldots \ldots \ldots \ldots \ldots$ & 147 & $-0.041 \pm 0.002$ \\
Flaccomio et al. (1999) & 236 & $0.070 \pm 0.003$ & 236 & $-0.072 \pm 0.008$ & 221 & $-0.147 \pm 0.007$ \\
Sung et al. (1997) & 117 & $-0.000 \pm 0.002$ & $\ldots \ldots \ldots \ldots$ & $\ldots \ldots \ldots \ldots \ldots$ & 116 & $0.001 \pm 0.010$ \\
\hline
\end{tabular}

\section{Time series analysis}

Our aim was to check all 10554 stars in the field for both periodic and irregular variability. For detecting these two types of variability we have used different techniques which are described in the following subsections.

\subsection{Periodic variables}

We used two periodogram analysis techniques to search for significant periodicity in the light curves of each of the 10554 monitored stars. Those stars which have fewer than 15 data points in their light curves were rejected from this analysis. $96 \%$ of the remaining 10503 stars have 80 or more data points in their light curves. In the following we describe the two used periodogram algorithms separately.

\subsubsection{The Scargle periodogram}

First we used the periodogram technique for unevenly sampled data described by Scargle (1982) and Horne \& Baliunas (1986) to search for significant periodicity in all monitored stars. The algorithm calculates the normalised power $P_{\mathrm{N}}(\omega)$ for a given angular frequency $\omega=2 \pi v$ and identifies the location of the highest peak in the calculated periodogram of each star. Finally, the light curve of each star is phased to the period according to the frequency of the highest peak. In order to decide whether there is a significant periodic signal of this period in the light curve, the height of this peak has to be related with a false alarm probability (FAP) which is the probability that a peak of this height is due simply to statistical variations, i.e. noise.

The standard procedure for determining the FAP is to use simulated light curves created with Monte Carlo simulations. It is necessary that the time sampling of the simulated light curves is identical with that of the measured light curves. The simulated light curves represent non-variable stars and therefore contain only a intrinsic scatter in the photometry, i.e. noise. For each simulated light curve the periodogram has to be calculated and the power of the highest peak in that periodogram has to be determined. After various simulations (typically $~ 10000$ ) the cumulative distribution of the power of the highest peak is used to determine the FAP: the FAP of a given power $P_{\mathrm{N}}$ is set to be the fraction of simulated non-variable stars which have a highest peak power that exceeds $P_{\mathrm{N}}$. If we have simulated for example 10000 light curves, the $1 \%$ FAP power $P_{\mathrm{FAP}=1 \%}$ is the power which was exceeded by the highest peak in 100 simulations.
The critical point in this procedure is the simulation of nonvariable or at least non-periodic stars. The simplest approach to doing this is to assume that the data points are statistically independent of each other. This is only true if the typical time scale for intrinsic variations of the sources is not larger than the (typical) time difference of the data points. Strictly speaking this assumption is not valid for our sample since the significant time scale for variations in PMS stars is $\sim 1 \mathrm{~d}$ (e.g. Herbst et al. 1994) and in some nights our data points were obtained at closely spaced intervals of much shorter length $(\sim 0.5 \mathrm{~h})$. Therefore we calculated the FAP using different types of simulations.

First we make the simplified assumption of uncorrelated data points and created light curves of pure noise using Monte Carlo simulations. The time sampling was chosen to be the same as in the real light curves. Gaussian distributed random variables with zero mean and standard deviation $\sigma$ were assigned to these dates. We set $\sigma=0.01$ mag since the mean standard deviation in the light curves of the (non-variable) comparison stars we used for calculating the relative light curves is of the same order. Therefore we expect a standard deviation of $\sigma=0.01$ in the light curve of a non-variable star. After 10000 simulations we calculated a $1 \%$ FAP power value of $P_{\mathrm{FAP}=1 \%}=10.2$ from the cumulative distribution of the highest peak as described above.

To allow for correlations between the data points a second type of simulations was carried out. Instead of simulating pure Gaussian noise, the measured light curves of the monitored stars were used. For the simulations we kept the time sampling the same as in the measured light curves. The relative magnitudes were randomly reassigned to the real dates. From the cumulative distribution of the maximum power we calculated a $1 \%$ FAP power value of $P_{\mathrm{FAP}=1 \%}=9.8$.

To be more conservative the highest power value of the two FAP simulations was used to define a cutoff level for the detection of periodic variables. Out of the 10554 analysed stars 1192 were brighter than $I_{\mathrm{C}} \leq 19.5 \mathrm{mag}$ and had a peak power $P_{\mathrm{N}} \geq 10.2$. As an example we show in Fig. 4 the complete light curve and the resulting phased light curve we found for four stars. In Fig. 5 we show a sample of phased light curves at different power levels.

\subsubsection{The CLEAN periodogram}

The Scargle periodogram technique makes no attempt to account for the observational window function $W(v)$, i.e. some 
Table 4. Photometric data, the complete table available electronically at the CDS. The column called "vari" lists the results of the time series analysis: pv refers to periodic and iv to irregular variables (see also the comments in the text). $\sigma$ is the standard deviation of the light curves from which outliers were rejected (see text). ptp is the estimated peak-to-peak variation in the light curves. Spectral type (SpT) were taken from Rebull et al. (2002), indicated by the index 1, or from Young (private communication), indicated by the index 2. The indentifications in the last column are the catalogue numbers of Y: Young (private communication), R: Rebull et al. (2002, private communication), S: Sung et al. (1997), P: Park et al. (2000), F: Flaccomio et al. (1999), FX: Flaccomio et al. (2000), W: Walker (1956), and LkH $\alpha$ Herbig (1954).

\begin{tabular}{|c|c|c|c|c|c|c|c|c|c|c|c|c|c|c|c|}
\hline Star & $\alpha(J 2000)$ & $\delta(J 2000)$ & $I_{\mathrm{C}}$ & err & $V-I_{\mathrm{C}}$ & err & $R_{\mathrm{C}}-I_{\mathrm{C}}$ & err & $R_{\mathrm{C}}-\mathrm{H} \alpha$ & err & vari & $\sigma$ & ptp & SpT name & cross id \\
\hline 6000 & $6: 41: 8.16$ & $9: 30: 4.4$ & 18.37 & 0.02 & & & 2.31 & 0.02 & -2.93 & 0.07 & iv & 0.073 & 0.26 & & \\
\hline 6001 & $6: 41: 8.19$ & $9: 36: 56.8$ & 16.91 & 0.01 & 4.06 & 0.06 & 2.06 & 0.01 & -3.32 & 0.02 & $\mathrm{pv}$ & 0.053 & 0.17 & & R3588 \\
\hline 6002 & $6: 41: 8.21$ & $9: 34: 9.9$ & 15.16 & 0.01 & 3.32 & 0.01 & 1.69 & 0.01 & -3.08 & 0.01 & & 0.016 & 0.05 & & R3591 \\
\hline 6003 & $6: 41: 8.21$ & $9: 38: 30.9$ & 16.56 & 0.01 & 3.83 & 0.08 & 1.93 & 0.01 & -2.88 & 0.04 & iv & 0.074 & 0.27 & & R3590 \\
\hline 6004 & $6: 41: 8.27$ & $9: 30: 23.4$ & 15.56 & 0.02 & 2.45 & 0.09 & 1.40 & 0.03 & -2.29 & 0.08 & iv & 0.105 & 0.35 & & \\
\hline 6005 & $6: 41: 8.28$ & $9: 38: 14.7$ & 13.10 & 0.01 & 0.73 & 0.01 & 0.35 & 0.01 & -3.38 & 0.01 & & 0.010 & 0.04 & $\mathrm{G}^{2} \mathrm{~V}^{1}$ & Y3607, R3592, W171 \\
\hline 6007 & 6:41:8.38 & $9: 40: 36.7$ & 16.10 & 0.01 & 2.64 & 0.01 & 1.48 & 0.01 & -3.13 & 0.01 & pv? & 0.015 & 0.06 & & R3594 \\
\hline 6008 & $6: 41: 8.43$ & $9: 37: 53.0$ & 16.70 & 0.01 & 3.45 & 0.03 & 2.03 & 0.01 & -2.95 & 0.13 & pv & 0.015 & 0.05 & & R3595 \\
\hline 6010 & $6: 41: 8.52$ & $9: 32: 51.5$ & 19.13 & 0.02 & 2.34 & 0.03 & 1.10 & 0.01 & -2.54 & 0.02 & $\mathrm{pv}$ ?/iv & 0.154 & 0.55 & & \\
\hline 6011 & $6: 41: 8.53$ & $9: 23: 6.6$ & 20.54 & 0.06 & 0.90 & 0.04 & 0.44 & 0.04 & & & & 0.126 & 0.52 & & \\
\hline 6012 & $6: 41: 8.55$ & $9: 31: 6.5$ & 18.02 & 0.03 & 4.00 & 0.14 & 2.42 & 0.04 & -3.36 & 0.16 & pv & 0.211 & 0.72 & & \\
\hline 6013 & $6: 41: 8.56$ & $9: 42: 52.1$ & 14.57 & 0.02 & 1.91 & 0.01 & 1.01 & 0.01 & -3.23 & 0.01 & pv & 0.062 & 0.18 & $\mathrm{~K} 7 \mathrm{~V}^{1}$ & Y3618, R3598, S312, FX110, W173 \\
\hline 6014 & $6: 41: 8.57$ & $9: 30: 4.2$ & 17.20 & 0.01 & 3.35 & 0.02 & 1.97 & 0.02 & -2.74 & 0.01 & pv & 0.016 & 0.05 & & \\
\hline 6015 & $6: 41: 8.58$ & $9: 28: 14.3$ & 19.94 & 0.06 & -0.50 & 0.04 & 0.00 & 0.05 & & & & 0.182 & 0.70 & & \\
\hline 6016 & $6: 41: 8.63$ & $9: 53: 47.3$ & 17.74 & 0.01 & 3.06 & 0.03 & 1.95 & 0.01 & -2.95 & 0.02 & pv? & 0.013 & 0.05 & & R3600 \\
\hline 6018 & $6: 41: 8.65$ & $9: 39: 11.9$ & 20.31 & 0.08 & & & 2.29 & 0.15 & & & & 0.056 & 0.21 & & \\
\hline 6019 & $6: 41: 8.65$ & 9:40:14.1 & 16.67 & 0.01 & 3.06 & 0.01 & 1.76 & 0.01 & -3.12 & 0.01 & pv & 0.010 & 0.04 & & R3599 \\
\hline 6020 & $6: 41: 8.70$ & $9: 53: 25.3$ & 20.28 & 0.06 & & & & & & & & 0.068 & 0.25 & & \\
\hline 6021 & $6: 41: 8.80$ & $9: 27: 54.0$ & 16.92 & 0.04 & -0.43 & 0.38 & 1.35 & 0.03 & -3.01 & 0.04 & & 0.058 & 0.24 & & \\
\hline 6022 & $6: 41: 8.81$ & $9: 23: 43.9$ & 12.91 & 0.02 & 1.83 & 0.02 & 0.98 & 0.02 & -3.13 & 0.02 & $\mathrm{pv}$ & 0.073 & 0.26 & $\mathrm{~K} 4 \mathrm{~V}^{1}$ & Y3650, R3602, P144, F381, FX111 \\
\hline 6023 & $6: 41: 8.84$ & 9:48:48.6 & 21.58 & 0.19 & & & & & & & & 0.331 & 1.18 & & \\
\hline 6024 & $6: 41: 8.84$ & $9: 53: 1.8$ & 15.38 & 0.03 & 2.44 & 0.02 & 1.34 & 0.02 & -2.81 & 0.03 & pv & 0.217 & 0.72 & & \\
\hline 6025 & $6: 41: 8.85$ & $9: 45: 28.0$ & 18.83 & 0.08 & & & 2.90 & 0.04 & -2.02 & 0.82 & & 0.021 & 0.07 & & R3603 \\
\hline 6026 & $6: 41: 8.85$ & $9: 52: 48.3$ & 20.57 & 0.18 & & & & & & & & 1.107 & 4.26 & & \\
\hline 6027 & $6: 41: 8.86$ & $9: 46: 1.6$ & 12.31 & 0.02 & 0.71 & 0.01 & 0.38 & 0.01 & -3.36 & 0.02 & & 0.014 & 0.05 & $\mathrm{G}^{5} \mathrm{~V}^{1}$ & Y3628, R3606 \\
\hline 6028 & $6: 41: 8.89$ & $9: 29: 45.7$ & 19.50 & 0.11 & 1.28 & 0.05 & 0.71 & 0.07 & & & & 0.238 & 1.07 & & \\
\hline 6029 & $6: 41: 8.90$ & 9:29:29.8 & 19.99 & 0.08 & 2.16 & 0.09 & 0.82 & 0.04 & & & & 0.108 & 0.46 & & \\
\hline 6030 & $6: 41: 8.92$ & $9: 29: 22.6$ & 19.59 & 0.06 & 2.45 & 0.14 & 1.12 & 0.05 & & & & 0.151 & 0.70 & & \\
\hline
\end{tabular}



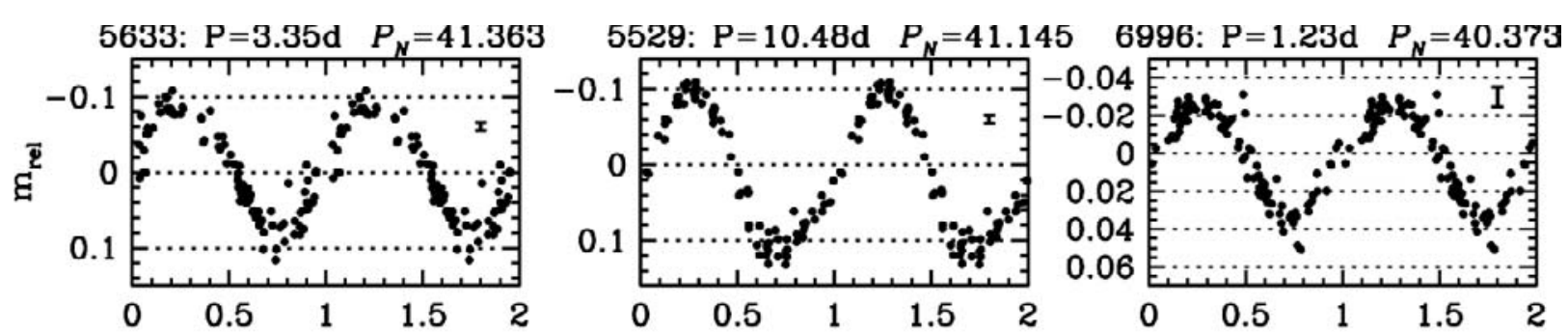

5320: $\mathrm{P}=8.48 \mathrm{~d} \quad P_{4}=42.161$
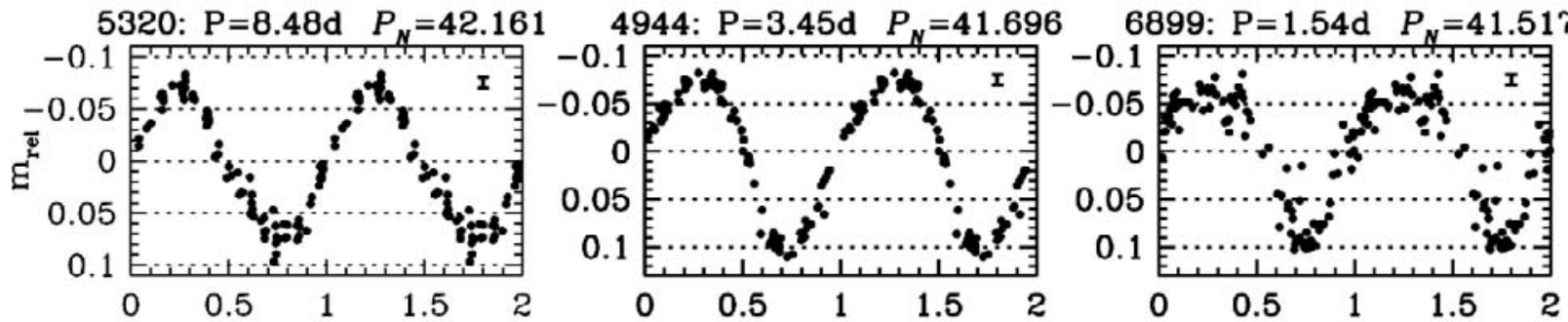

3383: $\mathrm{P}=1.10 \mathrm{~d} \quad P_{N}=28.979$
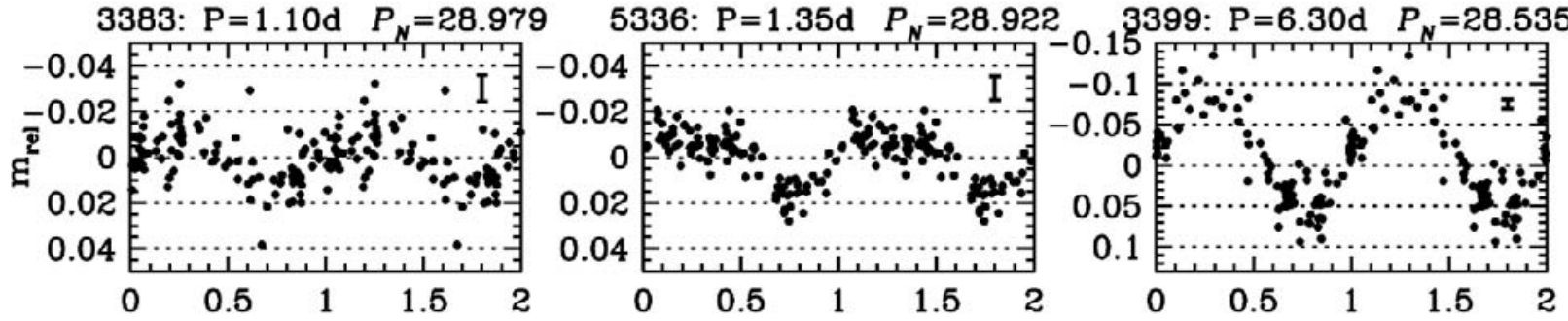

5190: $\mathrm{P}=1.88 \mathrm{~d} \quad P_{N}=28.42$
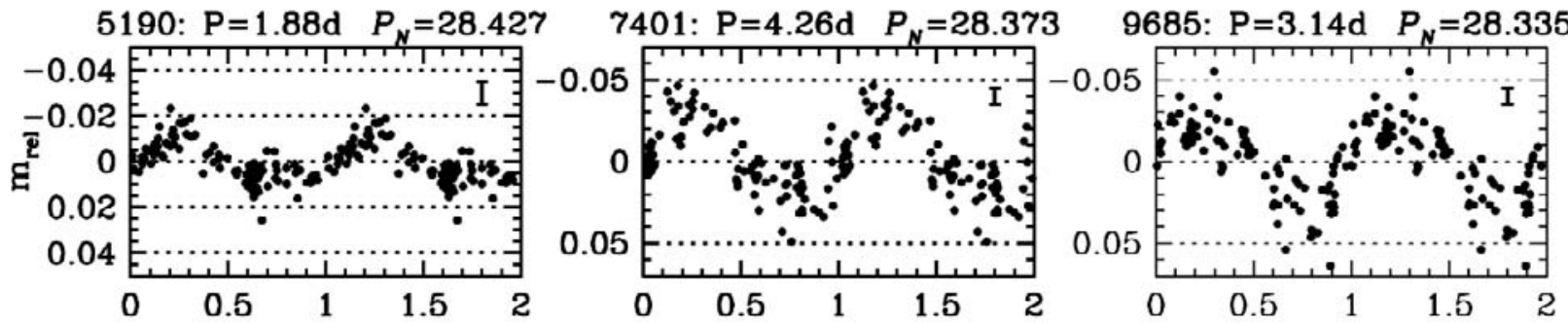

5408: $\mathrm{P}=5.15 \mathrm{~d} \quad P_{N}=13.979 \quad$ 5969: $\mathrm{P}=1.73 \mathrm{~d} \quad P_{N}=13.074$
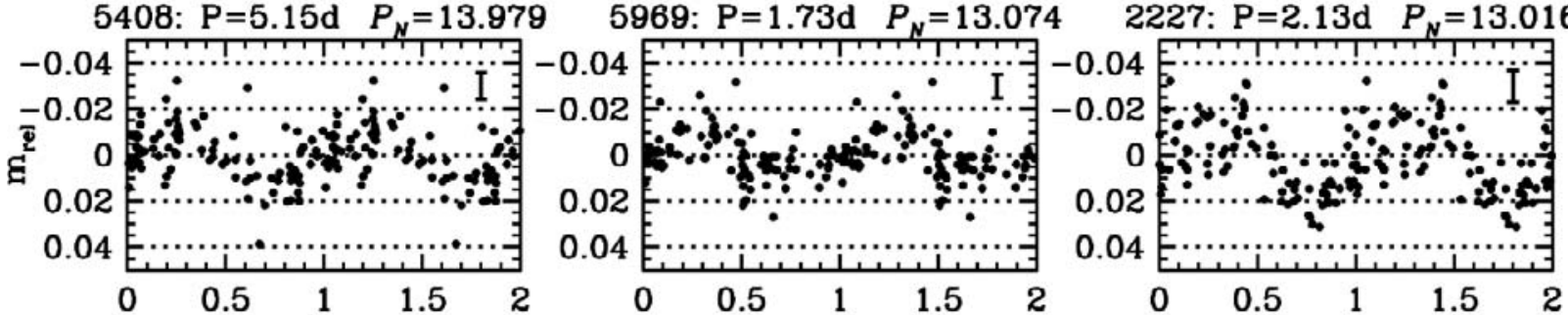

6019: $\mathrm{P}=0.64 \mathrm{~d} \quad P_{N}=12.368 \quad$ 5721: $\mathrm{P}=0.29 \mathrm{~d} \quad P_{N}=12.100$
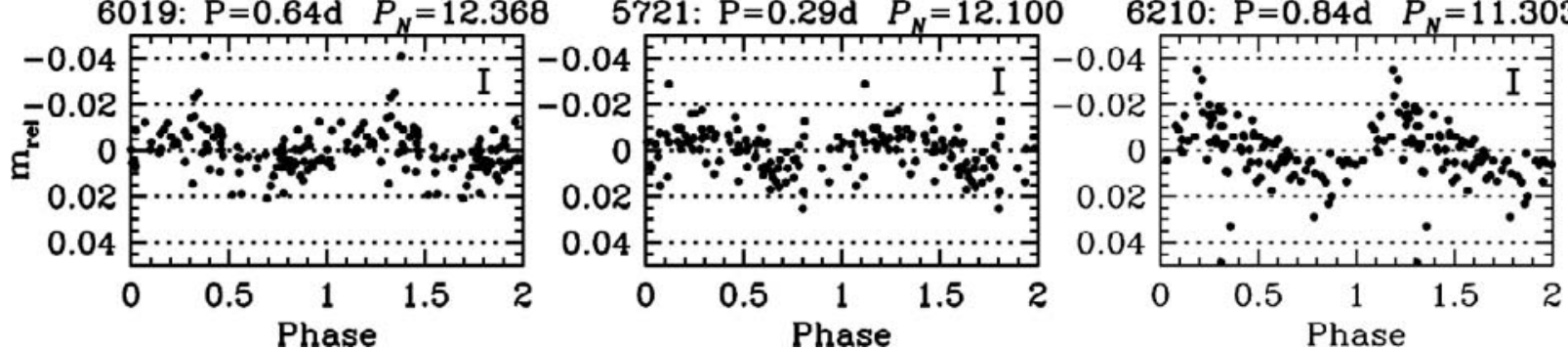

Fig. 5. Examples for phased light curves using the period $P_{\text {Scargle }}$ found by the Scargle periodogram technique. The peak power $P_{N}$ in the periodograms of these examples decreases from the upper left to the lower right panel. The examples in the two top rows represent the highest power values $\left(P_{N} \simeq 40, \ldots, 42\right)$ we found, the examples in the two middle rows represent median power values $\left(P_{N} \simeq 28\right)$ and the lower two rows represent the lowest power values we accepted $\left(P_{N} \simeq 11, \ldots, 14\right)$. The peak power $P_{N}$, the period $P_{\text {Scargle }}$ and the star's identification number is given for each example. The error bar in the upper right corner of each panel indicates the mean photometric error in the light curves. 
Table 5. Limiting magnitudes for a signal-to-noise ratio of 20 and 50 of observations in each of the filters $V, R_{\mathrm{C}}$ and $I_{\mathrm{C}}$. Also listed is the estimated limit for completeness in each filter.

\begin{tabular}{ccc}
\hline \hline Filter & Limiting & Magnitude \\
& $20 \sigma$ & $50 \sigma$ \\
\hline$V$ & 22.1 & 21.1 \\
$R_{\mathrm{C}}$ & 21.5 & 20.4 \\
$I_{\mathrm{C}}$ & 20.7 & 19.6 \\
\hline
\end{tabular}

of the peaks in the Scargle periodograms are normally a result of the data sampling. This effect is called aliasing and even the highest peak could be an artifact. The CLEAN periodogram technique by Roberts et al. (1987) tries to overcome this shortcoming of the Scargle periodogram. The observed power spectrum $|D(v)|^{2}$ is given by the convolution of the (complex) Fourier transformation $F(v)$ of the continuous signal from the star and the spectral window function $W(v)$ of the data set:

$D(v) \equiv F(v) \otimes W(v)$

(for a detailed description see Roberts et al. 1987 or Bailer-Jones \& Mundt 2001).

Even if the spectral window function is known it is not possible to de-convolve $D(v)$ directly. Therefore Roberts et al. (1987) modified the CLEAN algorithm that is known from the reconstruction of two-dimensional images from interferometric data. Based on their publicly available Fortran code we have created a $C$ realization of the CLEAN algorithm. A description of this algorithm is given in Appendix A. We calculated the CLEAN periodogram for each of the 10554 stars. After we had identified the highest peak in each of the periodograms the light curves of all stars were phased with the corresponding period $P_{\text {CLEAN. }}$

\subsubsection{The error in the measured periods}

The uncertainty in a measured period is set by two fundamental limits. First, it is related to the finite frequency resolution $\delta v$ of the power spectrum which makes it impossible to distinguish between closely separated periods and the uncertainty in a period is $\delta v / 2$. For a discrete data set the resolution $\delta v$ is given by the width of the main peak of the window function $W(v)$. If the time sampling is not too non-uniform this is related to the total time span $T$ of the observations with $\delta v \simeq 1 / T$ (Roberts et al. 1987). Since for small errors $\delta v / \delta P \simeq \mathrm{d} v / \mathrm{d} p=1 / P^{2}$ the error in the period is given by

$\delta P \simeq \frac{\delta v P^{2}}{2}$.

For larger errors (i.e. larger periods) this is only a lower limit on the period error. Second, for very short periods when the finite time span between the data points is comparable to the period the uncertainty in a period is given by the sampling error which is related to the typical spacing between the data points. Both of these fundamental limits leave their footprints in the main peak of the window function.
Since our time sampling is not very uniform (see Fig. 2) we determined the error of the periods directly from the width of this peak and for each star separately. The full width at half maximum of the main peak is typically (i.e. for a light curve with 88 data points) $v_{F W H M}=0.02601 /$ day which is 1.63 times larger than what we would expect from the total duration of the observations. This can be explained by the non-uniform sampling. For a light curve with 88 data points we finally assigned an error to the period $P$ which is given by $\delta P=0.026 \times P^{2}$. For light curves with less data points the factor differs depending on the width of the main peak of the window function.

\subsubsection{Final period determination}

For all of the 1192 stars that have a normalised peak power in the Scargle periodogram of $P_{N} \geq 10.2$ (i.e. FAP $\leq 1 \%$ ) both the light curve and the light curves phased with the periods found by both periodogram techniques were checked by eye and obvious wrong detections (e.g. due to light contamination from a close-by neighbour) were rejected.

The highest frequency which may be recovered from a sample with data points spaced at intervals of $\Delta$ is the Nyquist frequency $v_{\mathrm{N}}=1 /(2 \Delta)$ (Roberts et al. 1987). Using $\Delta=0.107$ day which is the median time difference of the data points in a light curve we therefore set a lower limit on the periods and accepted only periods with $P \geq 1 / \nu_{\mathrm{N}}=2 \Delta=0.21$ day.

In total we found 543 periodic variable stars. These stars are marked in Table 4 with a pv in the column "vari". For 520 $(95.8 \%)$ of these periodic variables the periods determined with the two different periodogram techniques agree within the estimated errors. In all cases where the periods determined with both methods did not agree the two periods were beat period of each other. For those stars we assigned one of the two periods to the star. We always choose the period for which the scatter in the phased light curve was lowest. For 17 of these 23 stars we assigned the CLEAN period but we kept the Scargle Period in six cases.

In addition all 543 periodic variables we found are listed in a separate Table 6 . In this table we give the periods we found with both the Scargle and the CLEAN periodogram techniques, the period we finally assigned to each star, and the estimated errors for this period. We also list the periods measured by Kearns \& Herbst (1998), if available. Out of the 543 stars 501 (91.9\%) have a peak power in the Scargle periodogram of $P_{N} \geq 12.40$ which corresponds to a FAP of $0.1 \%$.

The locations of the periodic variables in our observed field are shown in Fig. 7. Their distribution show that there are two concentrations of young stars in NGC 2264 which we call NGC $2264 \mathrm{~N}$ (north) and NGC $2264 \mathrm{~S}$ (south). These concentrations are already known in the literature: Sagar et al. (1988) report these two points of maximum stellar density of cluster members. This clustering agrees with the observed distribution of molecular gas, $\mathrm{H} \alpha$-emission stars and early type stars in the cluster (Mathieu 1986 and references therein). The stars located in these two concentrations will be used in Sect. 5.1 to determine the region in the colour-magnitude diagram where the PMS stars are located. 
Table 6. All periodic variables we found in our field. Listed are the identification number, the mean $I_{\mathrm{C}}$ magnitude, and the different periods detected by the Scargle periodogram technique $\left(P_{\mathrm{Sc}}\right)$, and the CLEAN periodogram technique $\left(P_{\mathrm{CL}}\right)$. Also shown is the period which we adopted in the end and the corresponding error for this period. Those periodic variables that passed both of our PMS criteria are marked with a " $y$ " in the column "PMS member". Those stars that failed at least one of the two tests are marked with a " $n$ " in the appropriate column. In the latter case the superscript indicates which test the star did not pass: $a$ ) rejected because of the location in $I_{\mathrm{C}}$ vs. $\left(R_{\mathrm{C}}-I_{\mathrm{C}}\right)$ diagram and $b$ ) rejected because of the $\left(R_{\mathrm{C}}-\mathrm{H} \alpha\right)$ colour. The full table is available electronically at the CDS.

\begin{tabular}{rccrrrrr}
\hline \hline Star & \multicolumn{1}{c}{$I_{\mathrm{C}}$} & (err) & \multicolumn{1}{c}{$P_{\mathrm{Sc}}$} & \multicolumn{1}{c}{$P_{\mathrm{CL}}$} & adopted $P$ & $\delta P$ & PMS \\
\hline 6001 & 16.91 & 0.01 & 6.01 & 5.98 & 6.01 & 0.50 & $\mathrm{n}^{b}$ \\
6008 & 16.70 & 0.01 & 1.32 & 1.32 & 1.32 & 0.02 & $\mathrm{y}$ \\
6012 & 18.02 & 0.03 & 4.35 & 4.33 & 4.35 & 0.26 & $\mathrm{n}^{b}$ \\
6013 & 14.57 & 0.02 & 10.48 & 10.46 & 10.48 & 1.43 & $\mathrm{y}$ \\
6014 & 17.20 & 0.01 & 1.59 & 1.59 & 1.59 & 0.03 & $\mathrm{y}$ \\
6019 & 16.67 & 0.01 & 0.65 & 0.64 & 0.65 & 0.01 & $\mathrm{y}$ \\
6022 & 12.91 & 0.02 & 1.73 & 1.73 & 1.73 & 0.05 & $\mathrm{y}$ \\
6024 & 15.38 & 0.03 & 9.71 & 9.81 & 9.71 & 1.23 & $\mathrm{y}$ \\
6031 & 13.85 & 0.01 & 3.92 & 3.90 & 3.92 & 0.20 & $\mathrm{y}$ \\
6032 & 14.72 & 0.01 & 0.80 & 0.80 & 0.80 & 0.01 & $\mathrm{y}$ \\
6039 & 14.44 & 0.01 & 5.83 & 5.76 & 5.83 & 0.44 & $\mathrm{y}$ \\
6042 & 16.91 & 0.01 & 0.54 & 0.54 & 0.54 & 0.00 & $\mathrm{y}$ \\
6043 & 15.01 & 0.01 & 10.77 & 10.64 & 10.77 & 1.51 & $\mathrm{y}$ \\
6045 & 17.19 & 0.01 & 1.75 & 1.76 & 1.75 & 0.04 & $\mathrm{y}$ \\
6055 & 13.64 & 0.01 & 5.92 & 5.98 & 5.92 & 0.47 & $\mathrm{y}$ \\
6063 & 15.33 & 0.01 & 3.38 & 3.38 & 3.38 & 0.15 & $\mathrm{y}$ \\
6064 & 16.13 & 0.01 & 2.18 & 2.17 & 2.18 & 0.07 & $\mathrm{y}$ \\
6067 & 12.78 & 0.01 & 2.93 & 2.92 & 2.93 & 0.14 & $\mathrm{y}$ \\
6077 & 19.77 & 0.03 & 2.40 & 2.37 & 2.40 & 0.08 & $\mathrm{y}$ \\
6079 & 15.83 & 0.01 & 5.22 & 5.23 & 5.22 & 0.35 & $\mathrm{y}$ \\
6081 & 15.07 & 0.01 & 0.68 & 0.68 & 0.68 & 0.01 & $\mathrm{y}$ \\
6093 & 16.53 & 0.01 & 0.96 & 0.96 & 0.96 & 0.01 & $\mathrm{n}^{a, b}$ \\
6101 & 18.00 & 0.01 & 0.46 & 0.46 & 0.46 & 0.00 & $\mathrm{n}^{a}$ \\
6102 & 15.15 & 0.01 & 9.04 & 9.10 & 9.04 & 1.06 & $\mathrm{y}$ \\
6115 & 17.08 & 0.01 & 0.71 & 0.71 & 0.71 & 0.01 & $\mathrm{y}$ \\
\hline & & & & & & &
\end{tabular}

Out of the stars which we rejected after our visual inspection another 136 were classified as possible periodic variables. These stars are marked with a $p v$ ? in the column "vari" of Table 4 but are not listed in Table 6, because they are not used for any further analysis (see Paper II).

\subsection{Irregular variables}

Since many of the PMS stars in our study show nonperiodic brightness modulations with a peak-to-peak variation up to 1 mag we defined any variable star for which no period could be detected as an irregular variable.

We searched for this irregular variability in the light curves of all stars that are not periodically variable in the magnitude range of $I_{\mathrm{C}} \leq 19.5$ (which corresponds to $S / N \geq 50$ ) that have at least 20 data points. Stars with close neighbours were rejected from this sample because our fixed-size aperture could lead to seeing-dependent overlapping with a (bright) neighbour, and hence imitate variability. Since the impact of a faint neighbour is negligible we used a rejection criteria that is magnitude dependent. If two stars were separated by less than 2 '.5 from each other, both of them were rejected from the analysis if the magnitude difference between the two objects was less than 2.0 mag. If the magnitude difference of such two stars is larger than 2.0 mag only the fainter star was removed and the brighter stars was kept.

In total we analysed 5927 non-periodic stars. 90.6\% of these stars have more than 70 data points in the light curve. For detecting the irregular variables we used a $\chi^{2}$ test, in which we calculate the probability that the deviations in the light curve are consistent with the photometric errors, i.e. the probability that the star is not variable. Therefore we evaluate

$\chi^{2}=\sum_{j=1}^{N}\left(\frac{m_{\mathrm{rel}}(j)}{\delta m_{\mathrm{rel}}(j)}\right)^{2}$

where $m_{\text {rel }}(j)$ is the relative magnitude and $\delta m_{\text {rel }}(j)$ is the error of the $j$ th data point in the light curve of a star. The probability that the light curve of a non-variable star with $N$ data points results in a value for chi-square that exceeds the measured value $\chi^{2}$ is given by ${ }^{2}$ (Press et al. 1992)

$Q\left(\chi^{2} \mid N\right)=\frac{\Gamma\left(\frac{N-1}{2}, \frac{\chi^{2}}{2}\right)}{\Gamma\left(\frac{N-1}{2}\right)} \equiv \frac{\int_{\chi^{2} / 2}^{\infty} t^{(N-3) / 2} \mathrm{e}^{-t} \mathrm{~d} t}{\int_{0}^{\infty} t^{(N-3) / 2} \mathrm{e}^{-t} \mathrm{~d} t}$.

The probability $P_{\text {vari }}$ that the star is variable is therefore $P_{\text {vari }}=$ $1-Q\left(\chi^{2} \mid N\right)$.

\footnotetext{
${ }^{2}$ Since the time series has been mean subtracted the number of degrees of freedom is $N-1$.
} 


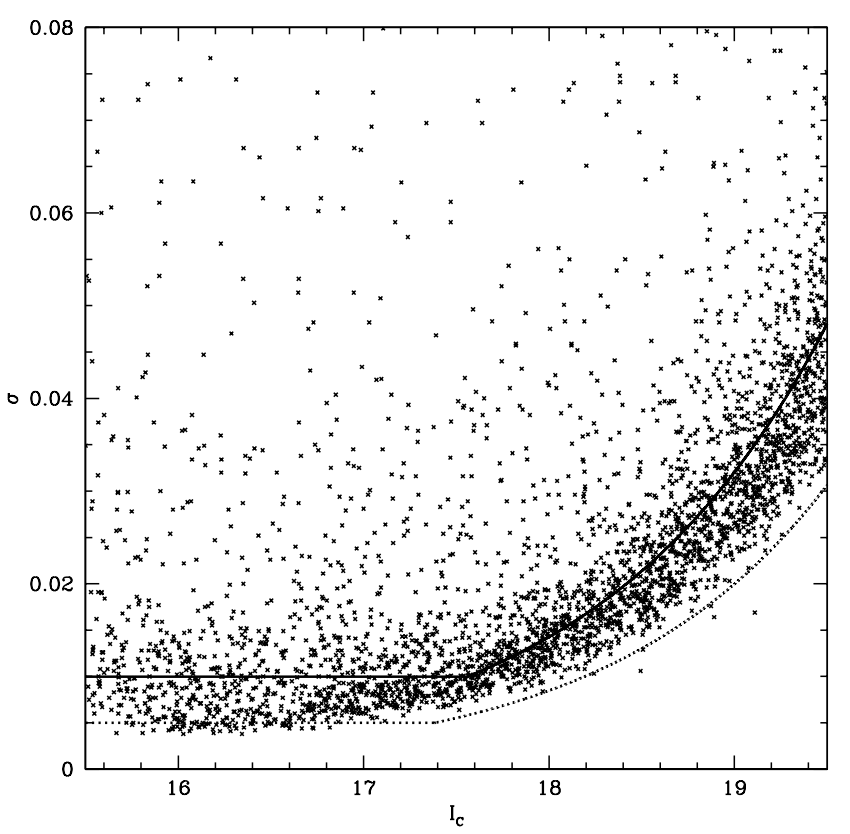

Fig. 6. Standard deviation $\sigma$ of all stars measured with the 500 s exposures as a function of magnitude. The solid line represents a fit $\left(S\left(I_{\mathrm{C}}\right)\right)$ to median values of $\sigma$. The dotted line shows the fit $\left(M\left(I_{\mathrm{C}}\right)\right)$ to the median of the mean photometric error $\overline{\delta m_{\text {rel }}}$ displayed in Fig. 3. The medians for both quantities were calculated in equally spaced magnitude bins.

We note that the $\chi^{2}$ test is very sensitive to an over- or underestimation of the errors. For example if the error in the single measurements is underestimated by a factor of 2 the value for $\chi^{2}$ is overestimated by a factor of 4 and we therefore deduce an overestimated probability $P_{\text {vari }}$ that the star is variable. In order to check whether the errors in the light curves are over- or underestimated we made a comparison of the scatter $\sigma$ and the average of the errors $\overline{\delta m_{\text {rel }}}=(1 / N) \sum_{j=1}^{N} \delta m_{\text {rel }}$ in the light curves, where $\delta m_{\text {rel }}$ is given by Eq. (4) in Bailer-Jones \& Mundt (2001). In Fig. 6 we show the scatter in the light curve of each star measured in the $500 \mathrm{~s}$ exposures. The dotted line is a fit $M\left(I_{\mathrm{C}}\right)$ to the median of the mean photometric errors of the $500 \mathrm{~s}$ exposures shown in the lower panel of Fig. 3. The solid line represents the fit $S\left(I_{\mathrm{C}}\right)$ of the median of the standard deviation $\sigma$ in the light curves. The median values for both the standard deviation $\sigma$ and the mean error $\overline{\delta m_{\text {rel }}}$ were calculated in different $I_{\mathrm{C}}$ magnitude bins. In both cases we fitted an exponential function in the fainter regime and a constant in the brighter regime.

It is evident that the estimated errors $\delta m_{\text {rel }}$ are systematically underestimated for the $500 \mathrm{~s}$ exposures and the same is the case for the $5 \mathrm{~s}$ and $50 \mathrm{~s}$. Therefore we corrected the errors measured for each star by calculating the ratio of the two functions for each of the three exposure times to give a correction function $C\left(I_{\mathrm{C}}\right)=S\left(I_{\mathrm{C}}\right) / M\left(I_{\mathrm{C}}\right)$. The error of each data point in the light curves of a given star was corrected using the equation

$\delta m_{\text {rel,cor }}(j)=C\left(I_{\mathrm{C}}\right) \delta m_{\mathrm{rel}}(j)$

where we used the mean magnitude for $I_{\mathrm{C}}$ of the star calculated as described in Sect. 3.2. By correcting the errors in this way we ensure that the relative distribution of the errors in a given light curve is conserved.

We note that we used a conservative fit for the standard deviation in the sense that the standard deviation may be overestimated by our fit (see Fig. 6). Since the same is the case for the fit $M\left(I_{\mathrm{C}}\right)$ of the mean errors this effect is minimised (but maybe not completely canceled) for the ratio $C\left(I_{\mathrm{C}}\right)=S\left(I_{\mathrm{C}}\right) / M\left(I_{\mathrm{C}}\right)$. However, a conservative estimate of the true error reduces the false detections of the $\chi^{2}$-test. On the other hand the test is less sensitive for low amplitude variations.

From Eq. (3) it is clear that a single outlier in the light curve (e.g. caused by a cosmic ray) could produce a very high value for the $\chi^{2}$. Since we are looking only for persistent variability we have applied a sigma clipping algorithm to the light curves before the $\chi^{2}$-test was performed. This algorithm uses the standard deviation $\sigma$ of a given light curve and removes all data points from this light curve that are located more than $2.5 \sigma$ above or below zero (note that the mean value was subtracted from the light curves).

After all stars had been analysed we found that many of the detected variable stars are located in the corners of our field. The light curves of these stars looked very similar and were well correlated with the changing seeing. A possible explanation for this is that the pixel scale of the WFI is different in the corners of the field. A similar result was recently found by Koch et al. (private communication) for the WFI. This leads to variations in the photometry of the stars located in the corners because the flux in the aperture of these stars changes with the seeing in a different way from the flux in the apertures of the comparison stars (the latter are not located in the corners, see Sect. 3.1). Therefore non-variable stars can mimic variability. In order to avoid this problem resulting from the variable pixel scale we performed the relative photometry again in exactly the same way as described in Sect. 3.1 but we used this time a significantly larger aperture diameter of 20 pixels (4".76) instead of 8 pixels. In this way we made sure that much more of the star's light falls into the aperture and the measurement is much less dependent on the seeing, i.e. the photometric errors dominate the changes due to seeing variations. The errors in the relative magnitudes were corrected in the same way as described above.

In order to decide whether any star in the field is variable we used the results of the $\chi^{2}$-tests based on the measurements with both aperture sizes. Only the stars that have a probability of $P_{\text {vari }} \geq 99.9 \%$ in both tests were assumed to be irregular variable. We used both aperture sizes and not only the measurements with the bigger aperture because with the large aperture we have increased the probability that the measurement of the stellar brightness is contaminated by the light of a closeby neighbour and the star therefore can mimic variability with the changing seeing. This effect is minimised for the photometry with the smaller aperture radius. On the other hand stars that do mimic variability because of the variable pixel scale do not pass the $\chi^{2}$-tests based on the measurements with a bigger aperture radius. Hence, a star that passes both tests is not affected by the variable pixel scale and light contamination from a close-by neighbour and therefore the variability is intrinsic to the star. 
In total we found 484 irregular variables out of the 5927 stars we analysed. The irregular variables are listed in column "vari" of Table 4 as $i v$. The spatial positions of the irregular variables are shown in Fig. 7. We remind the reader that irregular variables are defined here as variables with $P_{\text {vari }} \geq$ $99.9 \%$ for which we could not find any significant period in our periodograms.

With the $\chi^{2}$ test we are able to detect variability for stars brighter than $I_{\mathrm{C}}=16.0 \mathrm{mag}$ if the standard deviation $\sigma$ in the light curves is larger than $0.02 \mathrm{mag}$. Since we used images with $500 \mathrm{~s}$ exposure time for stars fainter than $I_{\mathrm{C}} \leq 16.0 \mathrm{mag}$, the sensitivity of the $\chi^{2}$ test is somewhat better for stars with $16.0 \mathrm{mag} \leq I_{\mathrm{C}} \leq 17.25 \mathrm{mag}$. In this magnitude range we are able to detect variability for stars with $\sigma \geq 0.015$ mag. For stars with $I_{\mathrm{C}}=18 \mathrm{mag}$ we are able to detect variability if $\sigma \geq 0.03$ mag.

Only $48.3 \%$ of the periodic variables are variable according to our $\chi^{2}$-test. It is not surprising that not all periodic variables are variable according to this test because the periodogram analysis is much more sensitive to low amplitude variations than the $\chi^{2}$ test (see Sect. 6). Furthermore we have adopted relatively conservative errors for our $\chi^{2}$ test (see above). This decreases the sensitivity to small amplitude variations.

We note that the different pixel scale in the corners of the field has only weak or no effect on the results of our periodic variability study. Since the seeing changes randomly the amplitudes of this random variation contributes to all powers in the power spectrum and not only to a single peak. Only $\sim 5-10$ periodic variables are located in the affected regions of the field. However, the variations in the corners of the field might have caused us to miss detecting a small number periodic variables.

\section{PMS membership}

The periodic and irregular variables we found in our field are not necessarily all PMS stars, although variability is probably one of the best indicators for youthfulness. Nevertheless there will be a certain degree of contamination by non-PMS stars if one selects candidates on the basis of variability. Therefore we have to disentangle variable PMS stars from variable background or foreground stars. This was done in two steps, namely using first the $I_{\mathrm{C}}$ vs. $\left(R_{\mathrm{C}}-I_{\mathrm{C}}\right)$ colour-magnitude diagram $(\mathrm{CMD})$ and second the $\left(R_{\mathrm{C}}-\mathrm{H} \alpha\right)$ vs. $\left(R_{\mathrm{C}}-I_{\mathrm{C}}\right)$ colour-colour diagram. In the following subsections we describe the two discrimination procedures in detail. Variable stars which pass both of these tests will be likely PMS members of the cluster and for sake of simplicity will therefore be called PMS stars in the subsequent discussion. For a full confirmation of the PMS nature of these stars additional observations are necessary, like measurements of the Li I $26707-$ line equivalent width. We expect, however, that only a minor fraction of the stars which passed both of the tests are non-PMS stars.

\subsection{PMS test I: The colour-magnitude diagram and determination of the PMS region}

In a CMD the PMS stars are located in a region above the main sequence (MS). This is due to their larger stellar radii and

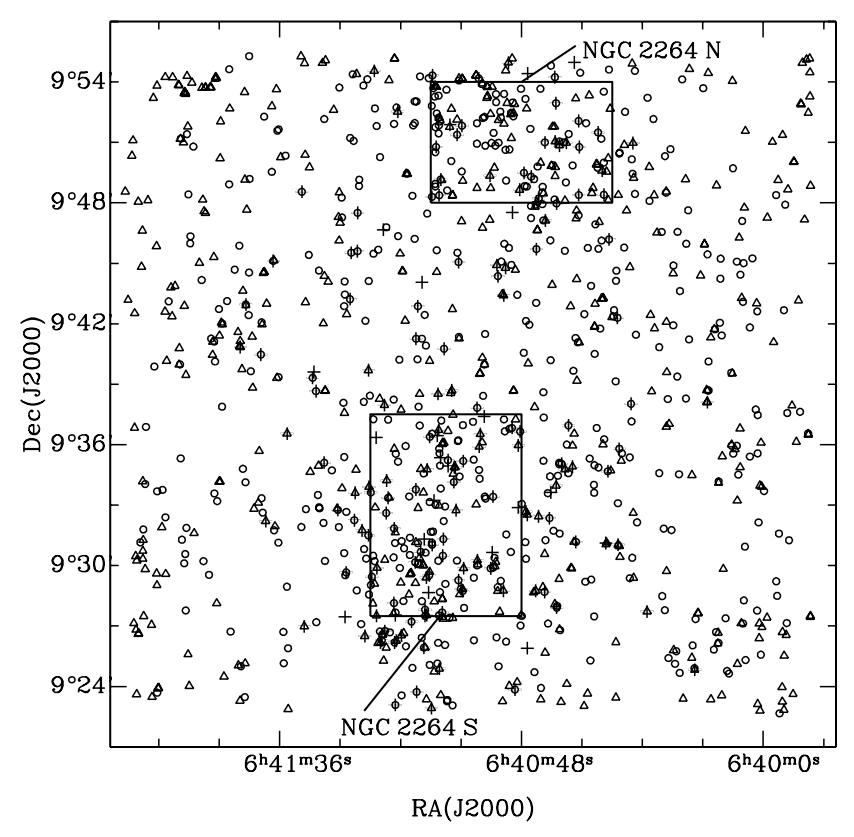

Fig. 7. The locations of all 543 periodic ( $(\circ)$ and 484 irregular $(\triangle)$ variables from our survey (including non-PMS stars). Those variable stars which were previously known as PMS stars are indicated by a vertical line $(\phi, \Delta$ respectively) while previously known PMS members which are not variable according to our investigations are marked by a cross (+). The two regions NGC $2264 \mathrm{~N} \& \mathrm{~S}$ with the highest concentration of variables are marked by boxes.

correspondingly larger brightness compared to MS stars of the same spectral type or colour. In order to determine the borders of the PMS region we placed a well defined sample of PMS stars in the CMD. This sample was selected in such a way that it is contaminated as little as possible by background and foreground stars. It consists of two subsamples: 1) previously known cluster members selected from the catalogues of Park et al. (2000) and Sung et al. (1997) and 2) a well selected subsample of the newly found periodic and irregular variables. The second subsample consists of our newly found periodic and irregular variables located in the two dense concentrations of variables in the cluster. These two concentrations are called NGC 2264 N \& S and are marked in Fig. 7. The two regions are a part of the so called "on cloud region" defined by Rebull et al. (2002) and as already mentioned above are known as regions of maximum stellar density in the cluster (Mathieu 1986, Sagar et al. 1988).

Determining the borders of the PMS region in the CMD only from the variable stars in NGC $2264 \mathrm{~N} \& \mathrm{~S}$ has the advantage of producing a maximum fraction of PMS stars relative to any background stars. This is due to high extinction of the dust located behind the cluster stars. This is shown by Fig. 11 which also illustrates that in particular for NGC $2264 \mathrm{~S}$ a very large background extinction is present. Since we selected only variable stars located in these regions the contamination of the this subsample with (non-variable) MS foreground stars is also negligible. Therefore the selected variable stars in the two regions NGC 2264 S and N are most likely PMS cluster members.

The CMD of all variable stars in NGC $2264 \mathrm{~N} \& \mathrm{~S}$ and all previously known PMS stars in our whole NGC 2264 field is 


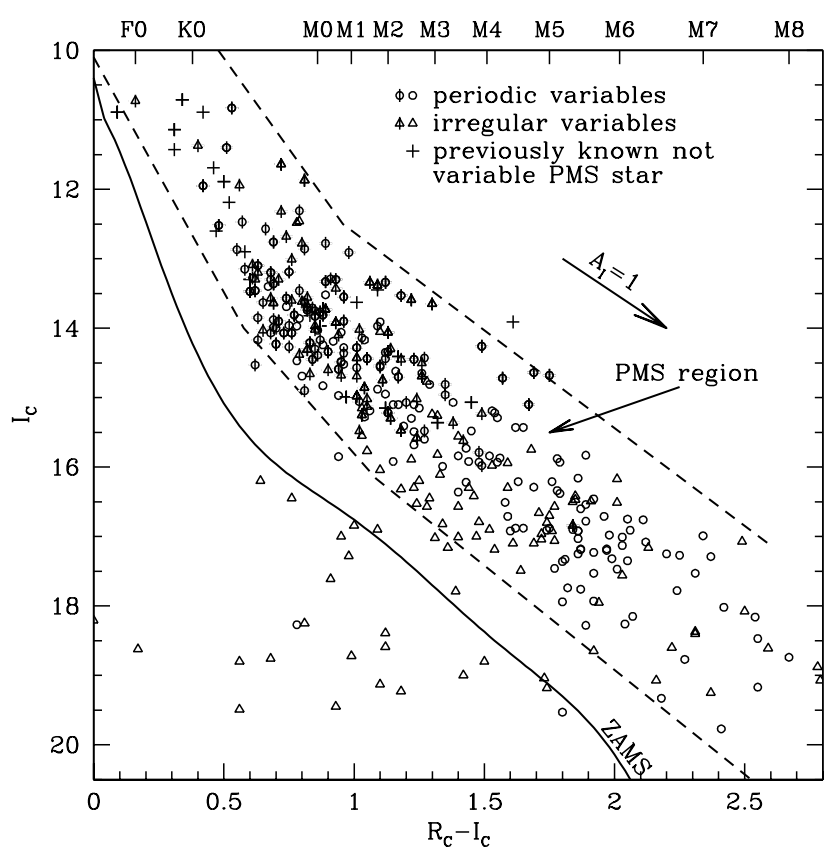

Fig. 8. Determination of the PMS region in the CMD. Only the variables in NGC 2264 S and N (see Fig. 7) and all previously known PMS stars in our whole NGC 2264 field are plotted. The symbols are the same as in Fig. 7. The two dashed lines indicate the lower and upper borders of the PMS region.

shown in Fig. 8. The spectral types marked at the top of the panel were determined by using photometric measurements of ZAMS stars with known spectral type. For stars with spectral types earlier than M0 mesurements reported by Johnson (1966) were used. For spectral types later than M0 measurements by Leggett (1992) and Kirkpatrick \& McCarthy (1994) were used. If necessary, transformation into the Cousins system were performed by applying the transformation equations by Bessel (1983). For the spectral types zero reddening is assumed. From this diagram we defined the PMS region in the CMD. The lower and upper borders of this region are indicated by the dashed lines.

The defined PMS region will obviously eliminate MS stars which are at the same distance as NGC 2264 or further away. Such stars are located in the CMD at or below the ZAMS indicated in Fig. 8 and are therefore well outside the PMS region. Because of their larger apparent $I_{\mathrm{C}}$ magnitude foreground MS stars with a distance between $\sim 300 \mathrm{pc}$ and $\sim 600 \mathrm{pc}$ could be located in the PMS region. However, as already mentioned above the contamination with these stars is probable negligible.

On the other hand it is not possible to discriminate between variable PMS stars and variable background giants using the PMS region in the CMD if the latter are at (large) distances which results in apparent magnitudes that shift them into the PMS region. K or M giants are typically between 3 and 10 magnitudes brighter than PMS stars at the same distance and the same spectral type. Therefore background giants with a corresponding smaller apparent $I_{\mathrm{C}}$ magnitude could be located in the PMS region of Fig. 8. Since the reddening vector is nearly parallel to the borders of the PMS region even a highly reddened giant will stay in the PMS region. In addition several

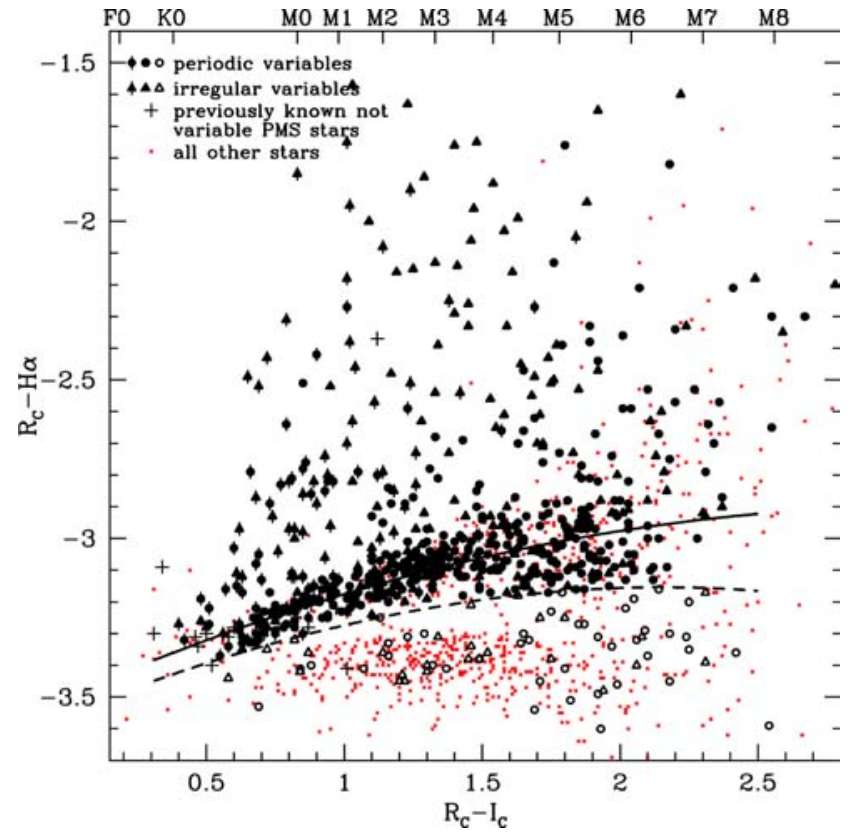

Fig. 9. The $\left(R_{\mathrm{C}}-\mathrm{H} \alpha\right)$ vs. $\left(R_{\mathrm{C}}-I_{\mathrm{C}}\right)$ colour-colour diagram for all stars which passed the first PMS test. Circles and triangles represent periodic and irregular variables respectively. Filled symbols indicate the stars which passed both PMS tests. Variables which were previously known as PMS are indicated by a vertical line. Non-variable previously known PMS stars are indicated by a cross. The solid line represents the fit of the locus of PMS and MS stars. Variable stars below the dashed line (open symbols) were rejected from the analysis because they are probable background giants.

types of giants are variable (e.g. RR Lyrae stars) and therefore it is necessary to eliminate these stars from our sample of variable stars using a different selection criterion. This will be done in Sect. 5.2

Of the 543 periodic variables we found in our whole NGC 2264 field, 451 stars were located in this PMS region and only these stars were used for the further analysis. These numbers together with the few (only 3) periodic variables outside the PMS region in Fig. 8 confirm that the regions NGC 2264 S \& N are very little contaminated with foreground or background MS stars compared to the rest of our field. That furthermore indicates that the above described procedure to define the PMS region in the CMD provides relatively reliable borders.

\subsection{PMS test II: The $\left(R_{C}-H \alpha\right)$ vs. $\left(R_{C}-I_{C}\right)$ colour-colour diagram}

As outlined in the previous section it is essential to eliminate background giants from the sample of variables we found. Therefore we used the location of the stars in the $\left(R_{\mathrm{C}}-\mathrm{H} \alpha\right)$ vs. $\left(R_{\mathrm{C}}-I_{\mathrm{C}}\right)$ colour-colour diagram (Fig. 9) as a second additional selection criterion for classifying a star as a PMS star; i.e. all stars which passed the first test have also to pass the second one to be classified as a PMS star. Many PMS stars, in particular CTTSs, show large $\mathrm{H} \alpha$ emission. Thus their $\left(R_{\mathrm{C}}-\mathrm{H} \alpha\right)$ colour is larger than the $\left(R_{\mathrm{C}}-\mathrm{H} \alpha\right)$ colour of a MS star of the 
Table 7. The relation between $\left(R_{\mathrm{C}}-I_{\mathrm{C}}\right)$ and $\left(R_{\mathrm{C}}-\mathrm{H} \alpha\right)$ colours for PMS/MS locus stars. The values for $\left(R_{\mathrm{C}}-\mathrm{H} \alpha\right)$ are calculated in bins of 0.05 magnitude width. $\sigma$ is the standard deviation of the $\left(R_{\mathrm{C}}-\mathrm{H} \alpha\right)$ colours in each bin.

\begin{tabular}{ccc|ccc|ccc|ccc}
\hline \hline$\left(R_{\mathrm{C}}-I_{\mathrm{C}}\right)$ & $\left(R_{\mathrm{C}}-\mathrm{H} \alpha\right)$ & $\sigma$ & $\left(R_{\mathrm{C}}-I_{\mathrm{C}}\right)$ & $\left(R_{\mathrm{C}}-\mathrm{H} \alpha\right)$ & $\sigma$ & $\left(R_{\mathrm{C}}-I_{\mathrm{C}}\right)$ & $\left(R_{\mathrm{C}}-\mathrm{H} \alpha\right)$ & $\sigma$ & $\left(R_{\mathrm{C}}-I_{\mathrm{C}}\right)$ & $\left(R_{\mathrm{C}}-\mathrm{H} \alpha\right)$ & $\sigma$ \\
\hline 0.38 & -3.36 & 0.05 & 0.83 & -3.22 & 0.06 & 1.28 & -3.10 & 0.08 & 1.73 & -3.02 & 0.12 \\
0.43 & -3.33 & 0.04 & 0.88 & -3.24 & 0.05 & 1.33 & -3.10 & 0.07 & 1.78 & -3.03 & 0.13 \\
0.48 & -3.33 & 0.05 & 0.93 & -3.22 & 0.06 & 1.38 & -3.08 & 0.08 & 1.83 & -2.99 & 0.15 \\
0.53 & -3.34 & 0.05 & 0.98 & -3.22 & 0.05 & 1.43 & -3.09 & 0.07 & 1.88 & -3.02 & 0.11 \\
0.58 & -3.30 & 0.04 & 1.03 & -3.20 & 0.06 & 1.48 & -3.07 & 0.10 & 1.93 & -3.02 & 0.14 \\
0.63 & -3.30 & 0.06 & 1.08 & -3.17 & 0.06 & 1.53 & -3.07 & 0.10 & 1.98 & -2.92 & 0.15 \\
0.68 & -3.30 & 0.05 & 1.13 & -3.16 & 0.07 & 1.58 & -3.06 & 0.10 & 2.03 & -3.03 & 0.11 \\
0.73 & -3.26 & 0.04 & 1.18 & -3.13 & 0.07 & 1.63 & -3.04 & 0.10 & 2.08 & -2.89 & 0.13 \\
0.78 & -3.24 & 0.04 & 1.23 & -3.12 & 0.08 & 1.68 & -3.05 & 0.10 & 2.13 & -2.87 & 0.20 \\
\hline
\end{tabular}

same spectral type. There also exists WTTSs or "naked" TTSs with weak or no detectable $\mathrm{H} \alpha$ emission (e.g. see Appenzeller $\&$ Mundt 1989). The locus of these latter stars is similar to the MS in the $\left(R_{\mathrm{C}}-\mathrm{H} \alpha\right)$ vs. $\left(R_{\mathrm{C}}-I_{\mathrm{C}}\right)$ colour-colour diagram.

On the other hand giants with spectral types later than $\sim \mathrm{K} 3$ have smaller $\left(R_{\mathrm{C}}-\mathrm{H} \alpha\right)$ colours than MS stars of the same $\left(R_{\mathrm{C}}-I_{\mathrm{C}}\right)$ colour. The differences in $\left(R_{\mathrm{C}}-\mathrm{H} \alpha\right)$ between giants and PMS/MS stars result from stronger molecular bands in the giants' spectra causing different spectral energy distributions in the $R_{\mathrm{C}}$ band. In addition giants are typically at larger distances and are therefore highly reddened. Since reddening affects mainly the $\left(R_{\mathrm{C}}-I_{\mathrm{C}}\right)$ colour they are shifted further away from the MS (i.e. to the right) with increasing distance. This behaviour was confirmed by a simulated $\left(R_{\mathrm{C}}-\mathrm{H} \alpha\right)$ vs. $\left(R_{\mathrm{C}}-I_{\mathrm{C}}\right)$ colour-colour diagram with the help of standard star spectra of different spectral type and luminosity (i.e. by multiplying the filter transmission curves of the WFI with the spectral energy distribution of standard stars spectra).

Therefore it is possible to discriminate between (background) giants and PMS stars using their location in the $\left(R_{\mathrm{C}}-\mathrm{H} \alpha\right)$ vs. $\left(R_{\mathrm{C}}-I_{\mathrm{C}}\right)$ colour-colour diagram. We determined the median $\left(R_{\mathrm{C}}-\mathrm{H} \alpha\right)$ colour as a function of $\left(R_{\mathrm{C}}-I_{\mathrm{C}}\right)$ for the locus of PMS/MS stars using the data shown in Fig. 9. In Table 7 we list the median $\left(R_{\mathrm{C}}-\mathrm{H} \alpha\right)$ colour as a function of $\left(R_{\mathrm{C}}-I_{\mathrm{C}}\right)$ for the stars on the PMS/MS locus. These values were derived in different $\left(R_{\mathrm{C}}-I_{\mathrm{C}}\right)$ colour bins of 0.05 magnitude width. We have fitted a quadratic function to these medians and obtained a locus relation of the form

$\left(R_{\mathrm{C}}-\mathrm{H} \alpha\right)_{\text {locus }}=-0.06 \times\left(R_{\mathrm{C}}-I_{\mathrm{C}}\right)^{2}+0.38 \times\left(R_{\mathrm{C}}-I_{\mathrm{C}}\right)-3.50 .(6)$

This relation is shown in Fig. 9 as the solid line. As we will show in Sect. 6.2, the vertical distance of a star from this line measures the strength of the star's $\mathrm{H} \alpha$ emission.

In order to discriminate between PMS stars and background giants we defined a lower discrimination level shown in Fig. 9 as a dashed line. This line is given by $\left(R_{\mathrm{C}}-\mathrm{H} \alpha\right)_{\text {low }}-1.65 \times \delta$, where $\delta$ is a fit of the standard deviation in $\left(R_{\mathrm{C}}-\mathrm{H} \alpha\right)$ in each $\left(R_{\mathrm{C}}-I_{\mathrm{C}}\right)$ bin and is given by $\delta=\mathrm{e}^{0.60\left(R_{\mathrm{C}}-I_{\mathrm{C}}\right)-3.42}$. Assuming that the locus of stars in each bin are Gaussian distributed $95 \%$ of the locus stars are located above the lower discrimination level which therefore represents a lower envelope of the locus. Any variable star (periodic or irregular) which passed the first

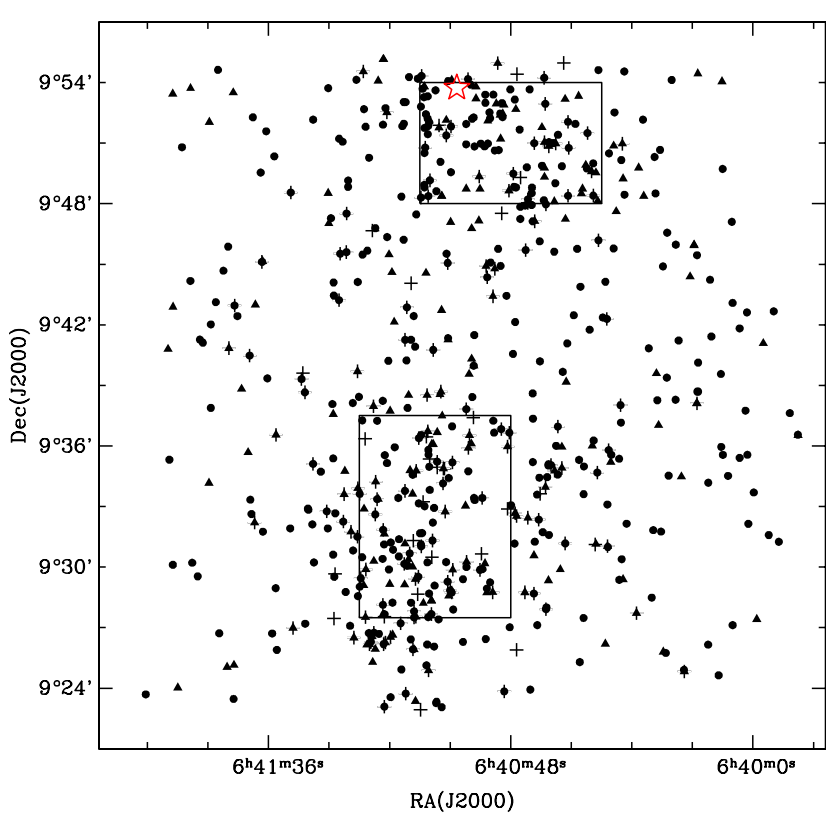

Fig. 10. Locations of all PMS variables from our survey which passed both tests including non-variable previously known PMS members. The symbols are the same as in Fig. 9. Also shown (indicated by the two boxes) are the two regions NGC $2264 \mathrm{~N} \& \mathrm{~S}$ which we used to determine the PMS region in the CMD. The star in the northern box indicates the location of the bright star S Mon ( $V=4.7 \mathrm{mag})$.

selection criterion described in Sect. 5.1 and that is located above this level was kept for the final analysis while stars below this level were rejected.

From the 451 periodic variables that passed the first selection criterion another 46 were rejected because of their $\left(R_{\mathrm{C}}-\mathrm{H} \alpha\right)$ colour. We finally have a total of 405 periodic and 184 irregular variables (with $P_{\text {vari }} \geq 99.9 \%$ ) which are all very likely PMS members of the NGC 2264 star forming region. Hence, we call these stars variable PMS members. The periodic variable PMS members are separately marked in Table 6.

\subsection{The spatial distribution of PMS members}

In Figs. 10 and 11 we show the spatial distribution of all PMS stars and the positions of non-cluster members, respectively. The latter were selected from the complete list of all 


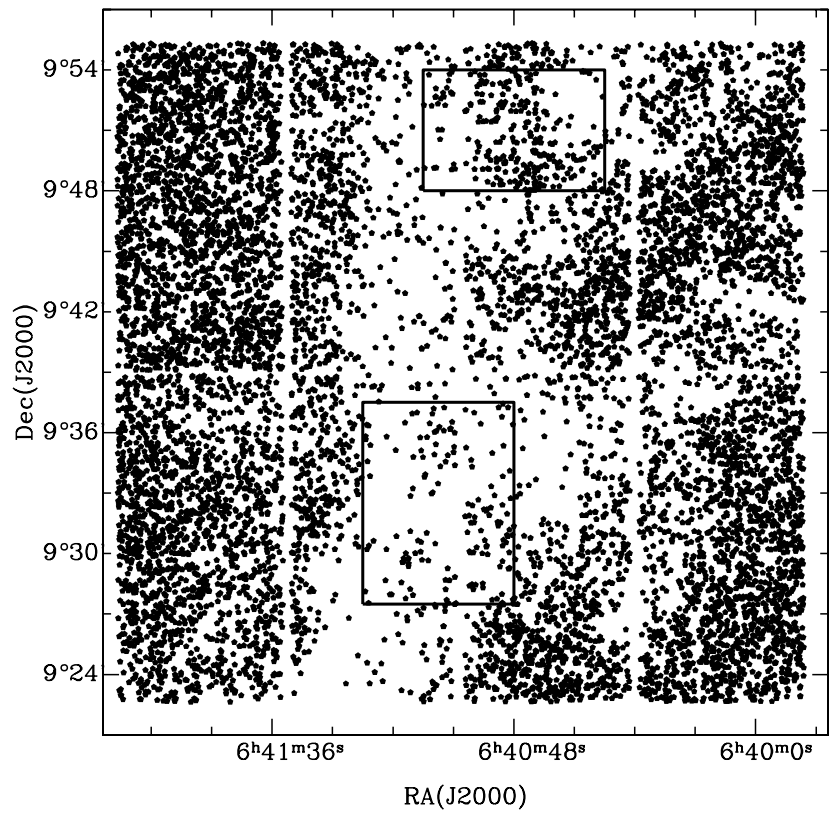

Fig. 11. Locations of all stars in the field that failed at least one of our PMS membership criteria (see Sect. 5). We also show the two regions which we used for determination of the PMS region in the CMD.

10554 stars in the field and represents all stars that failed at least one of our PMS membership criteria described in the previous sections. From the spatial distribution of the noncluster members one can clearly identify the extent of the dust cloud located towards NGC 2264. When comparing Fig. 10 with Fig. 11 it is evident that most PMS stars are located in the region with the highest background extinction, i.e. close to the dense gas and dust out of which they have probably been formed.

\subsection{Colour-magnitude diagrams of the periodic and irregular variables}

In Fig. 12 we show the $I_{\mathrm{C}}$ vs. $\left(R_{\mathrm{C}}-I_{\mathrm{C}}\right)$ colour-magnitude diagram of all periodic and irregular variables. It is evident that the periodic variable PMS members are much more concentrated around a line parallel to the ZAMS than the irregular variable PMS members. The reason for this could be a smaller age range of the periodic variables and/or a higher variability of the irregular variables compared to the periodic variables. In addition a higher intrinsic extinction of the irregular variables due to circumstellar disks could lead to a larger scatter. In the following section the differences between the two subsamples will be discussed in more detail.

In Fig. 12 we also show the $I_{\mathrm{C}}$ vs. $\left(R_{\mathrm{C}}-I_{\mathrm{C}}\right)$ colourmagnitude diagram of all (169) previously known PMS stars in NGC 2264 which we could identify in our sample. It is evident that the new PMS variables we found extend to much fainter magnitudes than the previously known PMS stars, i.e. we found new PMS stars mainly in the low mass regime which probably reaches down to the substellar limit of approximately $I_{\mathrm{C}}=18.5 \mathrm{mag}$ in NGC 2264 .



Fig. 12. The $I_{\mathrm{C}}$ vs. $\left(R_{\mathrm{C}}-I_{\mathrm{C}}\right)$ colour-magnitude diagram for different samples of stars. In a) we show all 543 periodic variables we found. The 405 periodic variables that passed our two PMS criteria are marked with filled circles while those periodic variables which did not pass at least one test are marked by open circles. The PMS zone is indicated by the dashed lines. The solid line represents the ZAMS sequence. Panel b) shows the CMD for the all 484 variable stars. The 184 irregular variables which fulfilled both of our PMS criteria are indicated by filled triangles. The 300 variable stars which failed at least one test are shown by open triangles. In panel c) we show the CMD of the previously known PMS stars in our whole NGC 2264 field.

\section{The nature of the variability}

In this section we investigate some general aspects of the stars' variability and investigate which differences in physical properties between periodic and irregular variables can be found.

\subsection{The degree of variability}

We use the standard deviation $\sigma$ of the light curves as an estimation of the degree of variability. The advantage of using $\sigma$ (see Sect. 4.2) rather than the peak-to-peak (ptp) variation of the stars for the characterisation of the variability is that $\sigma$ is more robust to outliers in the light curves (e.g. due to cosmic rays). In order to account for those statistical outliers in the light curves we have equated the ptp variation for each star with the magnitude difference between the third highest and the third lowest data point. Both quantities $\sigma$ and ptp variation are given in Table 4 for each star. We find that the ptp variation is typically three times the standard deviation but it can 


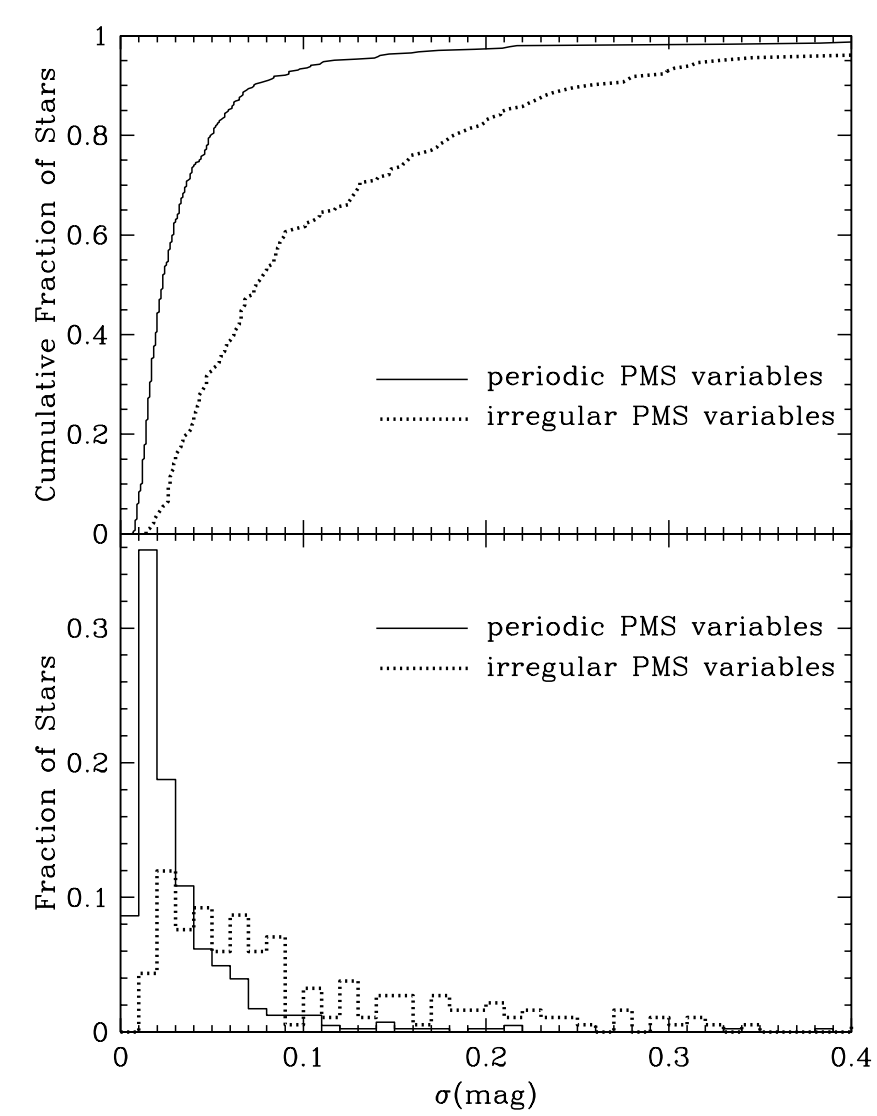

Fig. 13. a) The top panel shows the cumulative distribution of the standard deviation $\sigma$ for all 405 periodic and 184 irregular variable PMS stars respectively. b) The lower panel shows the histogram of $\sigma$ for the two different samples of stars in a).

vary between two and five times the standard deviation in some extreme cases.

In Fig. 13 we show the cumulative distribution of the standard deviation $\sigma$ for all 405 periodic and 184 irregular PMS variables. It is evident from this plot that about half of the periodic variables have $\sigma \leq 0.02$ mag while only $5.4 \%$ (10/184) irregular variables show this small level of variability. On the other hand only $2.7 \%$ of the periodic variables have a $\sigma \geq 0.2$ mag but $14.7 \%$ of the irregular variables exceed this level, i.e. the fraction of stars with a small $\sigma$ is always higher for the periodic variables.

This excess of periodic low amplitude stars is not surprising because the advantage of the periodogram analysis is that it is sensitive even for low amplitude variations. This results from the fact that the power from photometric errors (white noise) is distributed over the whole frequency domain of the power spectrum. Therefore even low amplitude variations close to (and below) the photometric errors can be detected. The $\chi^{2}$ test on the other hand is able to detect variability only if the variations in a light curve are inconsistent with the photometric errors. Our cutoff level of a probability $P_{\text {vari }}=99.9 \%$ corresponds to deviations of more than $3 \sigma$. Therefore we expect to find more periodic variables than irregular variables among the low amplitude variables.

In order to take any bias resulting from the different variability analysis methods into account we selected only those
264 periodic variables which are also variable according to the $\chi^{2}$ test and compared their distribution with that of the irregular variables. We found again that the two distributions are significantly different, as confirmed by a Kolmogorov-Smirnov test (Press et al. 1992) which shows that the probability that the two distributions are equivalent is less than $5 \times 10^{-15}$. A further discussion on this follows in Sects. 6.2 and 9.

A similar result was found in the ONC (Herbst et al. 2002) and in other T Tauri star associations. Herbst et al. (1994) have shown that most irregular variables are CTTSs and that they show large variations in their light curves. Periodic variables on the other hand are mainly WTTSs and have peak-to-peak variations in $I_{C}$ of less than $0.5 \mathrm{mag}$. This would correspond to standard deviations of $\sigma=0.1$ to $0.25 \mathrm{mag}$ for stars in our sample. Herbst et al. (2002) furthermore concluded that the variations in the stellar brightness of the periodic WTTSs are mainly caused by cool spots and the observed maximum peak-to-peak variation of $0.5 \mathrm{mag}$ is interpreted as the maximum possible brightness change which cool spots on the surface of a $\mathrm{K}$ or M star could cause. The periodic variables with peak-to-peak variations larger than $0.5 \mathrm{mag}$ are believed to be due to hot surface spots resulting from mass accretion. In our sample $3.5 \%$ of the periodic variables have peak-to-peak variations $\geq 0.5 \mathrm{mag}$ ( $\sigma \geq 0.16 \mathrm{mag}$ ). The irregular variability of CTTSs can have various reasons. Often it is attributed to variable mass accretion resulting in hot spots which are not stable in brightness, size, and location over a few rotation periods. In addition flare-like activities can be an additional source of variability. In reality the situation is probably more complicated and it could well be that some WTTSs have small hot spots and that CTTSs have cool spots in addition to large hot spots. However, for CTTSs it is much more difficult and in many cases even impossible to detect periodic brightness modulations caused by cold spots because of the overlying "noise" from irregular variability.

\subsection{The $\mathrm{H} \alpha$ emission index of periodic and irregular variables}

If the fraction of CTTSs is in fact higher among the irregular variables they should also show larger $\mathrm{H} \alpha$ emission than the periodic variables. In order to investigate the $\mathrm{H} \alpha$ emission of the stars in the two samples we defined a $\mathrm{H} \alpha$ emission index $\Delta\left(R_{\mathrm{C}}-\mathrm{H} \alpha\right)$ of a star by the following equation:

$\Delta\left(R_{\mathrm{C}}-\mathrm{H} \alpha\right)=\left(R_{\mathrm{C}}-\mathrm{H} \alpha\right)_{\text {star }}-\left(R_{\mathrm{C}}-\mathrm{H} \alpha\right)_{\text {locus }}$,

where the $\left(R_{\mathrm{C}}-\mathrm{H} \alpha\right)_{\text {locus }}$ is shown as a solid line in Fig. 9 and is given by Eq. (6). It is the $\left(R_{\mathrm{C}}-\mathrm{H} \alpha\right),\left(R_{\mathrm{C}}-I_{\mathrm{C}}\right)$ relation of a MS star. The $\mathrm{H} \alpha$ index is a measurement of the $\mathrm{H} \alpha$ emission. Using stars with known $\mathrm{H} \alpha$ equivalent width $\left(W_{\lambda}(\mathrm{H} \alpha)\right)$ we find (see Paper II) that $83 \%$ of the stars with an emission index of $\Delta\left(R_{\mathrm{C}}-\mathrm{H} \alpha\right) \geq 0.1 \mathrm{mag}$ have $W_{\lambda}(\mathrm{H} \alpha) \gtrsim 10 \AA$ and are therefore likely CTTSs. On the other hand about $85 \%$ of the stars with $\Delta\left(R_{\mathrm{C}}-\mathrm{H} \alpha\right)<0.1$ mag have $W_{\lambda}(\mathrm{H} \alpha)<10 \AA$ and are therefore most likely WTTS.

In Fig. 14 we show the cumulative distribution and the histogram of the $\mathrm{H} \alpha$ index for periodic and irregular variables. It is evident that the $\mathrm{H} \alpha$ emission index of the periodic variables is concentrated around zero and that only $22 \%$ of these 


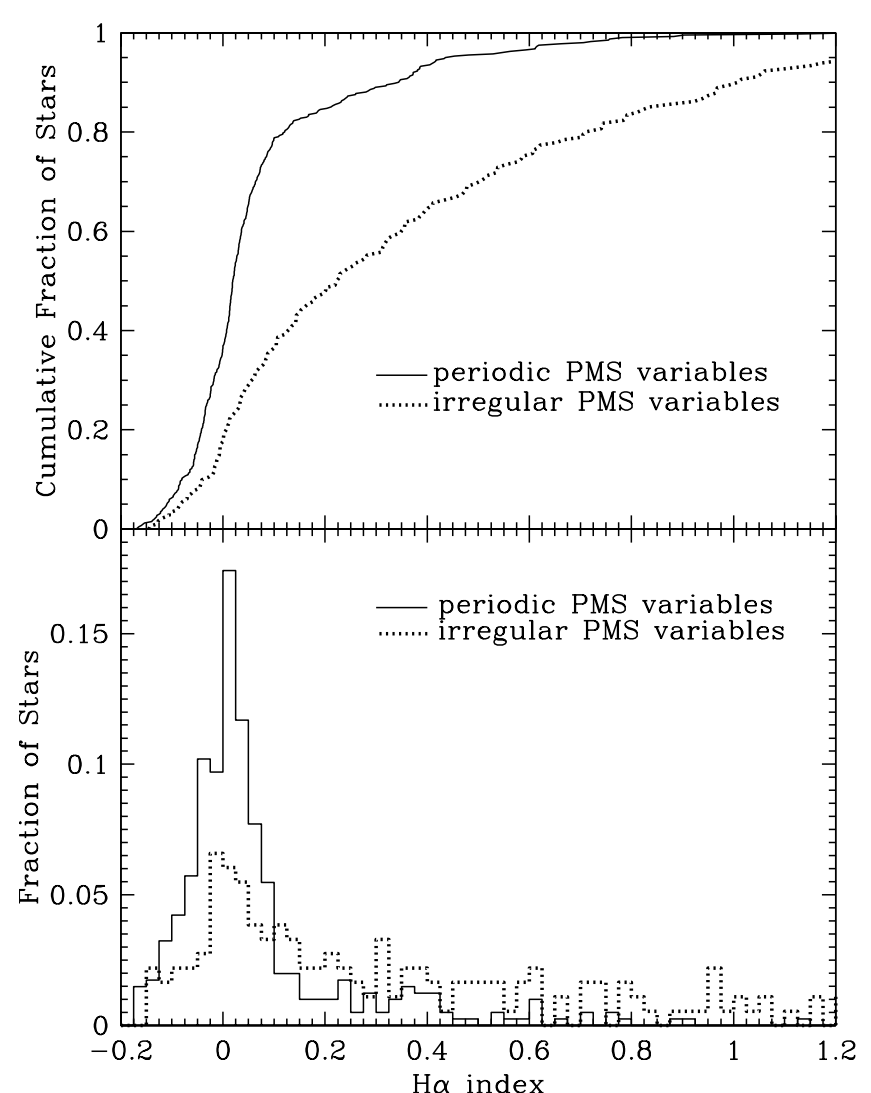

Fig. 14. a) The top panel shows the cumulative frequency distribution of the $\mathrm{H} \alpha$ index $\Delta\left(R_{\mathrm{C}}-\mathrm{H} \alpha\right)$ for all 405 periodic and 184 irregular variable PMS stars. b) The lower panel shows the histogram of the $\mathrm{H} \alpha$ index for the two different samples of stars in a).

stars exceed an $\mathrm{H} \alpha$ index of $0.1 \mathrm{mag}$. On the other hand the $\mathrm{H} \alpha$ emission index of the irregular variables is distributed over a larger range and for $68 \%$ of the irregular variables it is above the critical level of $0.1 \mathrm{mag}$.

This supports the interpretation that the fraction of CTTSs among the irregular variables is higher than the fraction of CTTSs among the periodic variables and vice versa for the WTTSs. In Sect. 9 we will quantify how strongly the sample of periodic variables is biased towards the WTTSs by comparing the fractions of CTTSs and WTTSs among the periodic variables with the expected fractions.

\subsection{The correlation between $\sigma$ and the $\mathrm{H}_{\alpha}$ index}

In order to investigate this interpretation further we look for a correlation between the degree of variability of the stars and their $\mathrm{H} \alpha$ index which is a measurement of their accretion and/or chromospheric activity. In Fig. 15 we show the $\mathrm{H} \alpha$ index and its median as a function of $\sigma$ for periodic and irregular variables respectively. It shows that for both samples there is an increase of the median $\mathrm{H} \alpha$ index with increasing $\sigma$. Each median was calculated in $\sigma$ bins of variable width containing 14 data points. Only the range $\sigma \leq 0.22 \mathrm{mag}$ is considered here since there are insufficient data points for larger values of $\sigma$. A Spearman rank-order correlation test (Press et al. 1992) indicates that the probability that $\sigma$ and the $\mathrm{H} \alpha$ index are not correlated is less



Fig. 15. The $\mathrm{H} \alpha$ index as a function of the degree of variability measured by the standard deviation $\sigma$ for all 405 periodic and 184 irregular variable PMS stars. Circles represent periodic variable stars while triangles represents irregular variables. The dashed and solid lines represent the medians of the $\mathrm{H} \alpha$ index for the irregular and periodic variables respectively in $\sigma$-bins of variable width with each containing 14 data points.

than $6 \times 10^{-28}$ if we use the joined sample of periodic and irregular variable stars. However, this high probability that there is a correlation between the two quantities is dominated by the periodic sample. If we use only the periodic sample the probability that $\sigma$ and the $\mathrm{H} \alpha$ index are not correlated is less than $9 \times 10^{-15}$. On the other hand the probability that $\sigma$ and the $\mathrm{H} \alpha$ index are correlated for the irregular variables is only 0.863 .

It is interesting that variability in the periodic variables is strongly correlated with the $\mathrm{H} \alpha$ index while there is no evidence for a correlation for the irregular variables. One possible reason is that the sizes and numbers of cool spots (i.e. the total spot area) on periodic variables (WTTSs) are correlated with the chromospheric activity as in other active late type stars, i.e. periodic variables with more spots have more active regions on their surface and therefore stronger chromospheric $\mathrm{H} \alpha$ emission. For the irregular variables we see that there is a large scatter in the $\mathrm{H} \alpha, \sigma$ relation, and even low amplitude variables have large $\mathrm{H} \alpha$ indices. This could imply that there are many CTTSs which have no large variations in their mass accretion rates. In addition these stars must have hot spot patterns which do not cause any large light modulations (e.g. accretion rings symmetric to the rotation axis, see e.g. Mahdavi \& Kenyon 1998).

\subsection{The $\left(V-R_{C}\right)$ vs. $\left(R_{C}-I_{C}\right)$ colour-colour-diagram}

In Fig. 16 we show the $\left(V-R_{\mathrm{C}}\right)$ vs. $\left(R_{\mathrm{C}}-I_{\mathrm{C}}\right)$ colour-colourdiagram for periodic and irregular variables which passed both of our two PMS tests. The stars with an $\mathrm{H} \alpha$-index of 


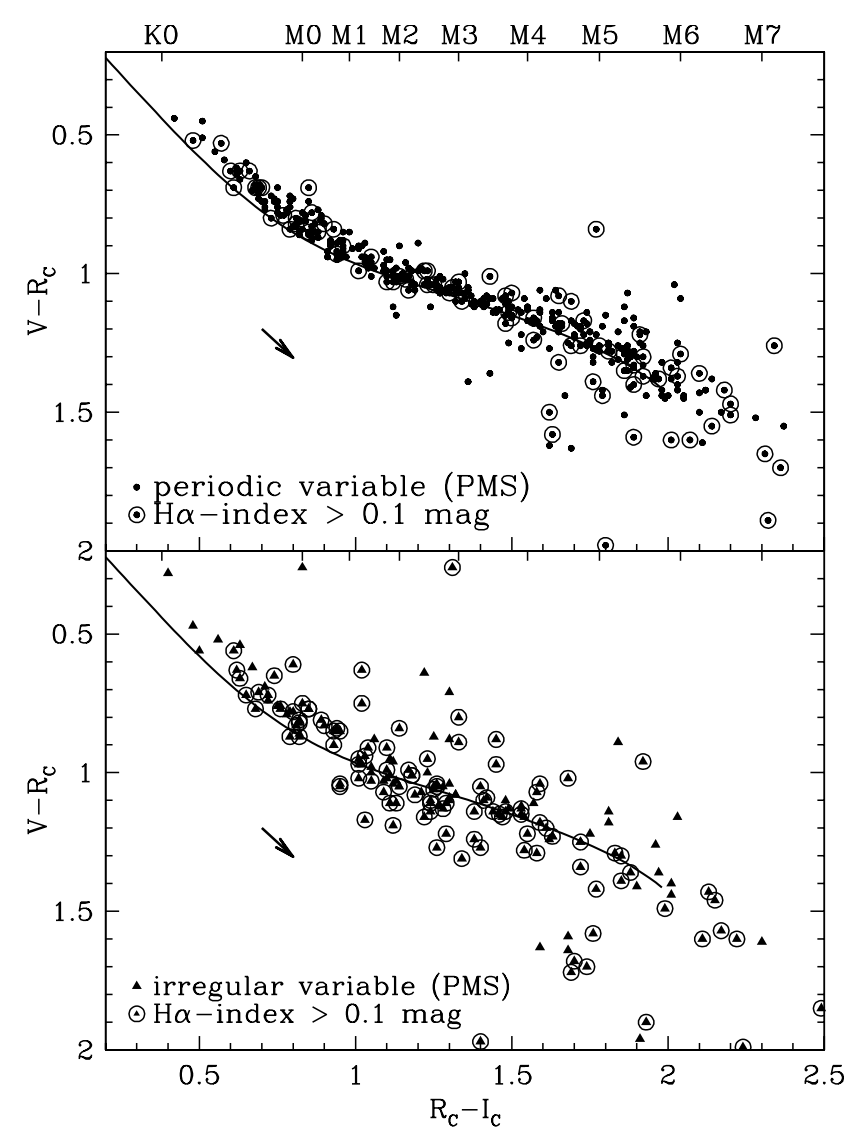

Fig. 16. The $\left(V-R_{\mathrm{C}}\right)$ vs. $\left(R_{\mathrm{C}}-I_{\mathrm{C}}\right)$ colour-colour-diagram for periodic (top panel) and irregular variables (bottom panel) that passed both of our two PMS tests. Those stars which have an $\mathrm{H} \alpha$-index $\geq 0.1$ and therefore show the properties of accreting CTTSs are marked with a surrounding circle. The solid lines in both panels represents the ZAMS. The arrow length and direction indicates the mean reddening towards NGC 2264.

$\Delta\left(R_{\mathrm{C}}-\mathrm{H} \alpha\right) \leq 0.1$ are marked separately. It is evident that the scatter is much larger among the irregular variables compared to the periodic variables. Agreement with the ZAMS is good, although deviations due to the smaller $\log g$ values of the PMS stars are expected. The data points below the MS (i.e. with larger $\left(V-R_{\mathrm{C}}\right)$ colours than a MS star) can be explained in both plots by embedded stars which are highly reddened with up to eight times the mean reddening. This interpretation is supported by the analysis of Rebull et al. (2002). They have determined an average $E\left(R_{\mathrm{C}}-I_{\mathrm{C}}\right)=0.1 \pm 0.02 \mathrm{mag}$ in NGC 2264, but showed that some stars in NGC 2264 are reddened by up to $E\left(R_{\mathrm{C}}-I_{\mathrm{C}}\right)=1 \mathrm{mag}$.

\section{7. "Non-variable" PMS members with strong $\mathrm{H} \alpha$ emission}

Based on our periodic and irregular variability study we have selected a total number of 584 PMS members (405 periodic and 184 irregular variables) using the two selection criteria described in Sect. 5. However, there may exist low-amplitude PMS stars, for which we could detect no significant variability. Reasons for a small degree of variability in WTTSs could be a more or less equally distributed pattern of many spots

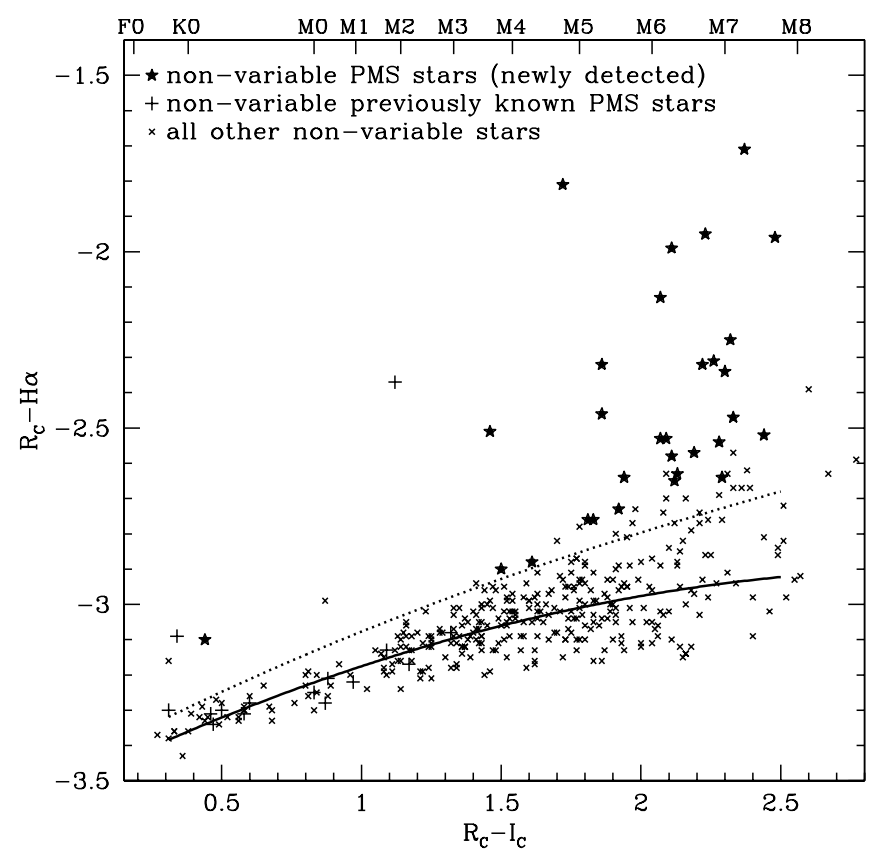

Fig. 17. The $\left(R_{\mathrm{C}}-\mathrm{H} \alpha\right)$ vs. $\left(R_{\mathrm{C}}-I_{\mathrm{C}}\right)$ colour-colour-diagram for all "non-variable" stars which passed the first PMS test (see Sect. 5) including previously known "non-variable" PMS members. The dotted line represents the modified second selection criterion for "nonvariable" PMS stars (see text). All "non-variable" stars which are located by more than their error in $\left(R_{\mathrm{C}}-\mathrm{H} \alpha\right)$ above this level are classified as PMS candidates.

causing only a small modulation of the stellar brightness and in CTTSs there could be phases of relatively stable mass accretion. To simplify matters we call these stars "non-variable" PMS stars. In order to search for "non-variable" PMS stars both PMS tests described in Sect. 5 were applied to all stars which were not detected to be variable. "Non-variable" PMS stars were selected if they passed both tests and have in addition strong $\mathrm{H} \alpha$ emission.

In Fig. 17 we show the $\left(R_{\mathrm{C}}-\mathrm{H} \alpha\right)$ vs. $\left(R_{\mathrm{C}}-I_{\mathrm{C}}\right)$ colourcolour-diagram for all of our non-variable stars which passed the first selection criterion. From these stars we selected only stars which showed an enhanced $\mathrm{H} \alpha$ emission using a more conservative threshold in the colour-colour diagram. This threshold is indicated by the dotted line in Fig. 17 which represents the upper envelope of the MS locus (solid line). The dotted line is given by

$\left(R_{\mathrm{C}}-\mathrm{H} \alpha\right)_{\text {high }}=\left(R_{\mathrm{C}}-\mathrm{H} \alpha\right)_{\text {locus }}+1.65 \times \delta$,

(for the definition of $\delta$ and $\left(R_{\mathrm{C}}-\mathrm{H} \alpha\right)_{\text {locus }}$ see Sect. 5.2). This upper discrimination level is equal to the lower discrimination level used in Sect. 5.2 mirrored at the PMS locus.

We consider only stars as "non-variable" PMS members if they are located more than their photometric error $\Delta_{\left(R_{\mathrm{C}}-\mathrm{H} \alpha\right)}$ above the upper discrimination line, i.e. $\left(R_{\mathrm{C}}-\mathrm{H} \alpha\right)-\Delta_{\left(R_{\mathrm{C}}-\mathrm{H} \alpha\right)} \geq$ $\left(R_{\mathrm{C}}-\mathrm{H} \alpha\right)_{\text {high }}$. In total 32 stars of the non-variables with $\left(R_{\mathrm{C}}-I_{\mathrm{C}}\right) \leq 2.5 \mathrm{mag}$ in Fig. 17 passed this additional PMS test. Out of these "non-variable" PMS members 2 stars (No. 5941 and No. 6164) were classified as PMS stars prior to this study. Another 12 previously known "non-variable" PMS members 
Table 8. New "non-variable" PMS stars in NGC 2264 selected using the PMS-test I and a slightly modified PMS-test II (see text). $P_{\text {vari }}$ is the probability that the star is variable according to our $\chi^{2}$ test. The other columns are the same as in Table 4.

\begin{tabular}{|c|c|c|c|c|c|c|c|c|c|c|c|c|c|c|}
\hline Star & $\alpha(J 2000)$ & $\delta(J 2000)$ & $I_{\mathrm{C}}$ & err & $V-I_{\mathrm{C}}$ & err & $R_{\mathrm{C}}-I_{\mathrm{C}}$ & err & $R_{\mathrm{C}}-\mathrm{H} \alpha$ & err & $\sigma$ & ptp & $P_{\text {vari }}$ & cross id \\
\hline 3130 & $6: 40: 21.32$ & $9: 48: 26.5$ & 19.00 & 0.03 & $\ldots \ldots$ & $\ldots$ & 2.26 & 0.03 & -2.31 & 0.10 & 0.040 & 0.17 & 0.0015 & \\
\hline 3455 & $6: 40: 26.95$ & $9: 49: 55.7$ & 18.28 & 0.02 & $\ldots \ldots$ & $\ldots$ & 2.19 & 0.02 & -2.57 & 0.05 & 0.014 & 0.05 & 32.8153 & $\mathrm{R} 2324$ \\
\hline 3646 & $6: 40: 29.41$ & $9: 47: 37.5$ & 16.83 & 0.02 & 3.26 & 0.06 & 2.07 & 0.02 & -2.13 & 0.07 & 0.017 & 0.05 & 99.3415 & $\mathrm{R} 2401$ \\
\hline 3648 & $6: 40: 29.42$ & $9: 41: 04.4$ & 18.60 & 0.01 & 3.39 & 0.07 & 2.07 & 0.03 & -2.53 & 0.06 & 0.017 & 0.06 & 0.0005 & \\
\hline 3680 & $6: 40: 29.89$ & $9: 50: 20.2$ & 16.79 & 0.01 & 2.62 & 0.01 & 1.50 & 0.01 & -2.90 & 0.01 & 0.007 & 0.03 & 0.0000 & \\
\hline 3840 & $6: 40: 31.92$ & $9: 50: 16.2$ & 19.42 & 0.03 & $\ldots \ldots$ & $\ldots$ & 2.28 & 0.05 & -2.54 & 0.15 & 0.034 & 0.12 & 1.6468 & \\
\hline 4323 & $6: 40: 38.30$ & $9: 29: 25.8$ & 17.71 & 0.04 & 3.06 & 0.02 & 1.81 & 0.01 & -2.76 & 0.05 & 0.157 & 0.65 & 0.0363 & \\
\hline 4877 & $6: 40: 45.89$ & $9: 39: 19.6$ & 19.19 & 0.04 & 2.07 & 0.08 & 2.09 & 0.09 & -2.53 & 0.18 & 0.035 & 0.13 & 0.0031 & \\
\hline 4924 & $6: 40: 46.68$ & $9: 48: 13.0$ & 16.92 & 0.01 & 3.05 & 0.02 & 1.86 & 0.02 & -2.46 & 0.02 & 0.007 & 0.03 & 0.0000 & R2959 \\
\hline 4960 & $6: 40: 47.17$ & $9: 28: 50.4$ & 11.45 & 0.01 & 0.88 & 0.01 & 0.44 & 0.01 & -3.10 & 0.06 & 0.014 & 0.06 & 0.0000 & Y2525, R2979, P67, MX, 114 \\
\hline 5456 & $6: 40: 54.74$ & $9: 54: 57.7$ & 19.23 & 0.02 & $\ldots$. & $\ldots$ & 2.23 & 0.07 & -1.95 & 0.44 & 0.034 & 0.17 & 0.7261 & \\
\hline 5567 & $6: 40: 56.64$ & $9: 32: 20.2$ & 19.33 & 0.03 & $\ldots \ldots$ & $\ldots$ & 2.30 & 0.05 & -2.34 & 0.16 & 0.024 & 0.10 & 0.0000 & \\
\hline 5570 & $6: 40: 56.73$ & $9: 38: 10.7$ & 18.98 & 0.02 & $\ldots \ldots$ & $\ldots$ & 2.48 & 0.07 & -1.96 & 0.10 & 0.031 & 0.14 & 59.4923 & \\
\hline 5627 & $6: 40: 59.39$ & $9: 31: 00.2$ & 17.60 & 0.02 & 3.14 & 0.09 & 1.86 & 0.02 & -2.32 & 0.03 & 0.022 & 0.07 & 99.3212 & \\
\hline 5631 & $6: 40: 59.53$ & $9: 34: 44.2$ & 19.23 & 0.02 & $\ldots \ldots$ & $\ldots$ & 2.22 & 0.05 & -2.32 & 0.09 & 0.044 & 0.16 & 3.4192 & \\
\hline 5982 & $6: 41: 07.56$ & $9: 41: 34.1$ & 16.07 & 0.01 & 2.53 & 0.04 & 1.46 & 0.01 & -2.51 & 0.10 & 0.014 & 0.05 & 2.5542 & $\mathrm{R} 3568$ \\
\hline 6047 & $6: 41: 09.23$ & $9: 29: 05.0$ & 18.48 & 0.02 & $\ldots \ldots$ & $\ldots$ & 2.11 & 0.03 & -1.99 & 0.06 & 0.019 & 0.07 & 0.2423 & \\
\hline 6169 & $6: 41: 13.11$ & $9: 24: 36.9$ & 16.44 & 0.01 & 2.79 & 0.01 & 1.61 & 0.01 & -2.88 & 0.01 & 0.006 & 0.03 & 0.0000 & Y3810, R3716, MX264 \\
\hline 6190 & $6: 41: 13.77$ & $9: 29: 32.5$ & 17.64 & 0.02 & 3.53 & 0.04 & 2.12 & 0.02 & -2.65 & 0.03 & 0.009 & 0.04 & 0.0394 & R3729 \\
\hline 6306 & $6: 41: 17.38$ & $9: 35: 56.5$ & 19.28 & 0.03 & $\ldots \ldots$ & $\ldots$ & 2.44 & 0.05 & -2.52 & 0.12 & 0.030 & 0.13 & 0.0551 & \\
\hline 6354 & $6: 41: 18.66$ & $9: 49: 02.7$ & 18.20 & 0.01 & 3.31 & 0.04 & 1.92 & 0.01 & -2.73 & 0.06 & 0.017 & 0.06 & 0.4781 & \\
\hline 6355 & $6: 41: 18.69$ & $9: 32: 52.7$ & 19.03 & 0.02 & $\ldots \ldots$ & $\ldots$ & 2.32 & 0.07 & -2.25 & 0.08 & 0.026 & 0.12 & 0.0000 & \\
\hline 6359 & $6: 41: 18.77$ & $9: 35: 19.6$ & 19.45 & 0.04 & $\ldots \ldots$ & $\ldots$ & 2.33 & 0.07 & -2.47 & 0.16 & 0.033 & 0.14 & 0.3219 & \\
\hline 6490 & $6: 41: 21.44$ & $9: 53: 01.9$ & 17.86 & 0.01 & 2.94 & 0.03 & 1.72 & 0.03 & -1.81 & 0.04 & 0.018 & 0.07 & 43.0053 & \\
\hline 6607 & $6: 41: 23.29$ & $9: 32: 30.7$ & 18.70 & 0.03 & 3.33 & 0.07 & 2.13 & 0.02 & -2.63 & 0.06 & 0.025 & 0.10 & 0.1210 & \\
\hline 6668 & $6: 41: 24.02$ & $9: 26: 53.0$ & 17.61 & 0.01 & 3.08 & 0.03 & 1.83 & 0.01 & -2.76 & 0.03 & 0.008 & 0.03 & 0.0082 & \\
\hline 6805 & $6: 41: 25.93$ & $9: 30: 26.6$ & 17.89 & 0.01 & 3.45 & 0.04 & 2.11 & 0.03 & -2.58 & 0.05 & 0.014 & 0.05 & 2.3883 & R4001 \\
\hline 7037 & $6: 41: 28.61$ & $9: 53: 58.5$ & 17.14 & 0.01 & 3.23 & 0.02 & 1.94 & 0.02 & -2.64 & 0.07 & 0.013 & 0.05 & 12.7790 & \\
\hline 7075 & $6: 41: 29.16$ & $9: 28: 48.6$ & 19.39 & 0.03 & $\ldots \ldots$ & $\ldots$ & 2.37 & 0.08 & -1.71 & 0.10 & 0.029 & 0.11 & 0.0000 & \\
\hline 10865 & $6: 42: 03.92$ & $9: 49: 02.2$ & 18.94 & 0.02 & 3.49 & 0.13 & 2.29 & 0.03 & -2.64 & 0.08 & 0.037 & 0.14 & 45.2014 & \\
\hline
\end{tabular}


did not pass this additional test. The 30 newly found stars are separately marked in Fig. 17 and listed in Table 8.

As one can see from Fig. 17 most $(83 \%)$ of the new "non-variable" PMS members have $\left(R_{\mathrm{C}}-I_{\mathrm{C}}\right) \leq 1.8$ mag. This can be understood if we consider that the photometric errors for these stars are typically larger compared to bluer (i.e. brighter) stars. Therefore any variations in the light curve are harder to detect.

In total we have identified 621 PMS stars in NGC 2264 including 405 periodic variable 184 irregular variable and 32 "non-variable" stars with relatively strong $\mathrm{H} \alpha$ emission. Compared to the 182 previously known PMS stars and PMS candidates reported by Park et al. 2000 (not including 26 previously known massive OB stars) we have increased the number of known PMS stars in NGC 2264 by a factor of 3.4. As already mentioned in Sect. 5.3 most of the newly found PMS stars are low mass stars $\left(M \lesssim 0.25 M_{\odot}\right)$ with masses probably extending into the substellar regime.

\section{Fraction of variable PMS stars and completeness level of our PMS sample}

In this section we estimate the fraction of variable PMS stars in the cluster. From this fraction we estimate the completeness level of our sample of variable PMS stars in NGC 2264. Therefore the central question in this section is: what fraction of PMS stars did we find through our photometric monitoring program and the adopted methods in selecting PMS stars?

The fraction of variable cluster members is given by $f_{\text {vari }}=$ $N_{\text {vari }} /\left(N_{\text {vari }}+N_{\text {non-vari }}\right)$, where $N_{\text {vari }}$ is the number of variable (both periodic and irregular) and $N_{\text {non-vari }}$ is the number of "non-variable" PMS stars in NGC 2264. These numbers (in particular $N_{\text {non-vari }}$ ) are well known for CTTSs since the PMS nature of these stars can be confirmed by an enhanced $\mathrm{H} \alpha$ emission. In addition we expect that there are also WTTSs (with no or weak $\mathrm{H} \alpha$ emission) which are "non-variable"; i.e. they have light modulations below our detection limit. Using the $I_{\mathrm{C}}$ vs. $\left(R_{\mathrm{C}}-I_{\mathrm{C}}\right)$ colour-magnitude or $\left(R_{\mathrm{C}}-\mathrm{H} \alpha\right)$ vs. $\left(R_{\mathrm{C}}-I_{\mathrm{C}}\right)$ colour-colour diagrams these stars are indistinguishable from MS foreground stars. In order to estimate the number of "non-variable" WTTSs we therefore have to keep the relative contamination by foreground MS stars as low as possible. This is achieved by using only the two regions NGC $2264 \mathrm{~N}$ and S (see Sect. 5.2) for our analysis rather than the whole observed field. In order to be not affected in the following analysis by photometric errors we restrict ourselves to stars with $I_{\mathrm{C}} \leq 18.0 \mathrm{mag}$ and $\left(R_{\mathrm{C}}-I_{\mathrm{C}}\right) \leq 1.8 \mathrm{mag}$.

In Table 9 we list the number of variable and "non-variable" stars in each of the two regions which passed both PMS tests (see Sect. 5) and are in these colour and magnitude ranges. From the last column of this table it is evident that in the two regions NGC $2264 \mathrm{~N}$ and S about $74 \%$ of the PMS candidates (i.e. stars that passed the two tests) are variable. About half of the PMS candidates show periodic light modulations while only one fourth of the PMS candidates are irregular variables. We note that these fractions are only lower limits since we assume that all stars in the two regions which passed both tests are indeed PMS stars. In particular we assume that the
Table 9. The estimated fraction of variable stars in NGC $2264 \mathrm{~N}$ and $\mathrm{S}$ with $I_{\mathrm{C}} \leq 18.0 \mathrm{mag}$ and $\left(R_{\mathrm{C}}-I_{\mathrm{C}}\right) \leq 2.0 \mathrm{mag}$. Listed are number and fractions of periodic PMS variables $\left(N_{\mathrm{p}-\text { vari }}\right)$, irregular PMS variables $\left(N_{\text {i-vari }}\right)$, "non-variable" PMS candidates $\left(N_{\text {non-vari }}\right)$, the total number of all PMS candidates $\left(N_{\text {PMS-total }}=N_{\text {p-vari }}+N_{\text {i-vari }}+N_{\text {non-vari }}\right)$, and the number and fractions of PMS variables $\left(N_{\mathrm{p}-\text { vari }}+N_{\mathrm{i} \text {-vari }}\right)$ in the two concentrations of PMS stars NGC $2264 \mathrm{~N}$ and S (for a definition of the PMS candidates see text). The fractions were calculated relative to the total number of PMS star candidates $\left(N_{\mathrm{PMS}-\text { total }}\right)$ in the two regions.

\begin{tabular}{lrrr}
\hline \hline Sample of stars & \multicolumn{1}{c}{ North } & \multicolumn{1}{c}{ South } & \multicolumn{1}{c}{ North \& South } \\
\hline$N_{\text {PMS-total }} \ldots \ldots$ & $110 \ldots \ldots$ & $120 \ldots \ldots$ & $230 \ldots \ldots \ldots$ \\
$N_{\text {non-vari }} \ldots \ldots$ & $25(22.7 \%)$ & $36(30.0 \%)$ & $61(26.5 \%)$ \\
$N_{\text {p-vari }} \ldots \ldots$ & $56(50.9 \%)$ & $54(45.0 \%)$ & $110(47.8 \%)$ \\
$N_{\text {i-vari }} \ldots \ldots$ & $29(26.4 \%)$ & $30(25.0 \%)$ & $59(25.7 \%)$ \\
\hline Variables $\ldots$. & $85(77.3 \%)$ & $84(70.0 \%)$ & $191(73.5 \%)$ \\
\hline
\end{tabular}

contamination by non-members in the variable sample is negligible; i.e. all variables that passed both PMS test are actually PMS stars.

However, if we further assume that the two regions are representative for the whole cluster we can conclude that at least $f_{\text {vari }}=74 \%$ of the PMS stars have been detected to be variable. This lower limit of the fraction of detected variable stars is not significantly higher if we consider only brighter stars; e.g. for stars with $I_{\mathrm{C}} \leq 16.0 \mathrm{mag}$ in NGC $2264 \mathrm{~N}$ and $\mathrm{S}$ we obtain a fraction of $f_{\text {vari }}=76 \%$.

Since we expect all PMS stars in the cluster to be variable at some level our variability study is complete at a $74 \%$ level for stars with $I_{\mathrm{C}} \leq 18.0 \mathrm{mag}$ and $\left(R_{\mathrm{C}}-I_{\mathrm{C}}\right) \leq 1.8 \mathrm{mag}$. As we will show in the following section the completeness level differs for WTTSs and CTTSs. While nearly all (95\%) of the CTTSs are found to be variable only $68 \%$ of the WTTSs are found to be variable.

In summary we conclude that our method of photometric monitoring is a very powerful tool for finding most PMS stars in our cluster since at least three quarters of the whole PMS population in NGC 2264 (with $\left(R_{\mathrm{C}}-I_{\mathrm{C}}\right) \leq 1.8 \mathrm{mag}$ ) could be identified this way. Therefore, our database of periodic and irregular variables should be a representative subset of the PMS stars in NGC 2264.

\section{Fractions of periodic variables among CTTSs and WTTSs}

In the following we try to estimate the fraction of periodic variables among the WTTSs and CTTSs in order to investigate how strongly the period distribution of NGC 2264 is biased by the non-detection of periods in these two groups of stars. Again we restrict our analysis to stars with $I_{\mathrm{C}} \leq 18.0 \mathrm{mag}$ and $\left(R_{\mathrm{C}}-I_{\mathrm{C}}\right) \leq 1.8 \mathrm{mag}$. Furthermore we consider in this section only stars that passed both PMS tests.

As already outlined in Sect. 6 the periodic variables are biased towards the WTTSs since it is much harder to detect periodic brightness modulations of CTTSs in the presence of a superimposed irregular variability. However, we will show below that this bias towards the WTTSs will be partially compensated 
by those WTTSs which have brightness modulations below our detection limit and have therefore not been found.

As in Sects. 6 and 7 we regard all stars with enhanced $\mathrm{H} \alpha$ emission measured by the $\mathrm{H} \alpha$ index as CTTSs. This is the case for $N_{\text {CTTS }}=145$ stars in our whole investigated region. While $89(61.4 \%)$ of these stars are irregularly variable only $49(33.8 \%)$ are periodically variable and $7(4.8 \%)$ are "non-variable". Thus only for one third (49/145) of the CTTSs periods are detectable.

It is more difficult to estimate the total number of WTTSs in the cluster $\left(N_{\text {WTTS }}\right)$ since "non-variables" WTTSs are indistinguishable from MS foreground stars in the $I_{\mathrm{C}}$ vs. $\left(R_{\mathrm{C}}-I_{\mathrm{C}}\right)$ colour-magnitude and in the $\left(R_{\mathrm{C}}-\mathrm{H} \alpha\right)$ vs. $\left(R_{\mathrm{C}}-I_{\mathrm{C}}\right)$ colour-colour diagram. However, with the results of the previous section we can relatively easily estimate the total number of PMS stars in the cluster $\left(N_{\mathrm{PMS}}\right)$ and since $N_{\mathrm{PMS}}=$ $N_{\text {CTTS }}+N_{\text {WTTS }}$ we can determine $N_{\text {WTTS }}$ indirectly.

Therefore we first estimate $N_{\text {PMS }}$. In total 465 stars with $\left(R_{\mathrm{C}}-I_{\mathrm{C}}\right) \leq 1.8 \mathrm{mag}$ and $I_{\mathrm{C}} \leq 18.0 \mathrm{mag}$ are periodic or irregular variables. Since at least $73.5 \%$ of the PMS stars are variable (see Sect. 8) we get $N_{\mathrm{PMS}}=465 / 0.735 \simeq 630$. With $N_{\text {WTTS }}=$ $N_{\text {PMS }}-N_{\text {CTTS }}$ we get $N_{\text {WTTS }}=630-145=485$ which is an upper limit since 0.735 is a lower limit.

Of the periodic variables with $\left(R_{\mathrm{C}}-I_{\mathrm{C}}\right) \leq 1.8 \mathrm{mag}$ and $I_{\mathrm{C}} \leq 18.0$ mag 265 have a small $\mathrm{H} \alpha$ index and are therefore classified as WTTSs. In addition 62 irregular variables are classified as WTTSs. Using these numbers we conclude that for at least 55\% (265/485) of the WTTSs in NGC 2264 we have detected a period and at least $13 \%(62 / 485)$ of the WTTSs are irregular variable; i.e. for $68 \%$ of the WTTSs we detected variability.

How do the measured period fractions of $55 \%$ and $34 \%$ for the WTTSs and CTTSs respectively influence the final period distribution? To answer this question let us first assume that the periods for all (estimated) 630 PMS stars in the cluster with $\left(R_{\mathrm{C}}-I_{\mathrm{C}}\right) \leq 1.8 \mathrm{mag}$ and $I_{\mathrm{C}} \leq 18.0$ mag could be measured. Using $N_{\text {CTTS }}=145$ and $N_{\text {WTTS }}=485$ (see above) we get that $23 \%(145 / 630)$ of the stars are CTTSs while the fraction of WTTSs is $77 \%(485 / 630)$. In our study $16 \%(49 / 341)$ of the periodic variables in these colour and magnitude ranges are CTTSs and $84 \%(265 / 314)$ of the periodic variables are WTTSs. From these numbers we conclude that the final period distribution is slightly biased towards WTTSs.

\section{Summary and conclusions}

We have carried out an extensive search for rotation periods and variability in NGC 2264. The main results of this investigation are as follows:

1. We have obtained absolute photometry in the Cousins $V$, $R_{\mathrm{C}}$, and $I_{\mathrm{C}}$ bands and instrumental $\mathrm{H} \alpha$ magnitudes for about 10600 stars with $9.8 \mathrm{mag} \leq I_{\mathrm{C}} \leq 21 \mathrm{mag}$ in NGC 2264 . Relative light curves with more than 80 data points each were obtained for each of these stars in the $I_{C}$ band. Two different periodogram analysis techniques and a $\chi^{2}$ test yielded a sample of 543 periodic and 484 irregular variables with 11.4 mag $\leq I_{C} \leq 19.7$ mag in the observed field.
2. In order to check the PMS nature of the periodic and irregular variables two different PMS selection criteria were applied. These criteria are their locations in the $I_{\mathrm{C}}$ vs. $\left(R_{\mathrm{C}}-I_{\mathrm{C}}\right)$ colour-magnitude diagram and their location in the $\left(R_{\mathrm{C}}-\mathrm{H} \alpha\right)$ vs. $\left(R_{\mathrm{C}}-I_{\mathrm{C}}\right)$ colour-colour diagram. In this way 405 periodic and 184 irregular variable PMS stars could be selected from the sample. This is an enormous increase in the number of known rotation periods compared to the about 30 published periods prior to this study. The rotation periods of the periodic variables are typically between a quarter of a day and 15 days.

3. Using an enhanced $\mathrm{H} \alpha$ emission index as a PMS indicator we could identify an additional 35 PMS stars which are "non-variable" according to our study. Together with the 589 variable PMS stars we found a total of 624 PMS stars in NGC 2264. Before our study only 182 PMS members and candidates were known. Most of our newly found PMS stars are fainter than $I_{\mathrm{C}} \simeq 15 \mathrm{mag}$ and of late spectral type $(\gtrsim \mathrm{M} 2)$.

4. Comparison of the distribution of the standard deviation $\sigma$ in the light curves as well as the distribution of the $\mathrm{H} \alpha$ emission index for periodic variables with the corresponding distributions for the irregular variables strongly suggests that our method for determining the rotation periods preferentially selects weak-line T Tauri stars (WTTSs) while classical T Tauri stars (CTTSs) are underrepresented. This interpretation is confirmed by the calculated fractions of periodic variables among WTTSs and CTTSs which, however, show that the bias of the measured rotation periods towards WTTSs is relatively small. We have estimated that $23 \%$ of the PMS stars in the cluster are CTTSs but only $15 \%$ of the periodic variables are CTTSs. The corresponding numbers for WTTS are $77 \%$ and $85 \%$ respectively.

5. The different properties of the two subclasses of $\mathrm{T}$ Tauri stars (CTTSs and WTTSs) are supposed to be due to the different mechanisms which produce the variability. While the variability of WTTSs is likely due to large (magnetically) cool spots the variability of CTTSs is believed to be mainly caused by hot spots resulting from accretion flows onto the star. On CTTSs the periodic brightness variations resulting from cool spots (which are believed to exist on their surface too) are often undetectable due to the "noise" from the irregular variability. Determining the rotational periods for a larger fraction of CTTSs by photometric monitoring would therefore require more extensive observations (e.g. larger time base and dense sampling).

6. We have estimated that we have detected variability among at least $70 \%$ of the PMS stars (defined according to our PMS tests) in NGC 2264 with $I_{\mathrm{C}} \leq 18.0 \mathrm{mag}$ and $\left(R_{\mathrm{C}}-I_{\mathrm{C}}\right) \leq 1.8 \mathrm{mag}$. From these numbers it is evident that extensive photometric monitoring with a similar accuracy as in our study is a powerful tool for finding most PMS stars. We emphasise that this method is highly efficient only if there is enough data with a proper sampling to allow a search for periodic variables because many PMS members were identified by their periodicity and not just by their variability. We conclude that the sample of variable PMS stars obtained in this study is a representative 
subset of the cluster members and it is not expected that additional monitoring programs will substantial increase the number of known PMS stars at least for stars with $I_{\mathrm{C}} \leq$ $18.0 \mathrm{mag}$.

Acknowledgements. We thank Luisa Rebull, Russ Makidon, and Steven Strom for providing their partly unpublished photometric data and their unpublished rotational data. We also thank Eric Young for providing unpublished spectral types. The authors thank Jochen Eislöffel for comments on the manuscript.

\section{Appendix A: The CLEAN algorithm}

The CLEAN algorithm we used in Sect. 4.1.2 subtracts the window function iteratively from the observed, or raw, spectrum in the following way. First the spectral window function

$$
W(v)=\frac{1}{N} \sum_{n=1}^{N} \mathrm{e}^{-2 \pi i v t_{n}}
$$

and the observed spectrum

$$
D(v)=\frac{1}{N} \sum_{n=1}^{N} d\left(t_{n}\right) \mathrm{e}^{-2 \pi i v t_{n}}
$$

are calculated at discrete frequencies with a spacing of $\Delta v=$ $1 /(10 T)$, where $T=t_{1}-t_{N}$ is the total length of the data span. In our case $T$ is about 62.8 days and the resolution of our CLEAN periodograms is therefore $\Delta v=0.0016 / d$.

The input into the $i$ th iteration is the spectrum $D_{i}$, where the iteration process starts with the (complex) raw spectrum $D_{i=1} \equiv D$. During each iteration step $i=1,2, \ldots, N$ the algorithm determines the frequency $v_{i}$ and the power $\left|D_{i}\left(v_{i}\right)\right|$ of the highest peak in the spectrum $D_{i}$. Assuming that this peak represents a true period in the signal the aliasing powers $A_{i}$ at other frequencies are calculated with

$$
A_{i}(v)=c_{i} W\left(v-v_{i}\right)+\left(c_{i}\right)^{*} W\left(v+v_{i}\right)
$$

where $c_{i}$ is the $i$ th complex clean component, that represents the amplitude of the peak and is defined as

$$
c_{i}=g \frac{D_{i}\left(v_{i}\right)-D_{i}^{*}\left(v_{i}\right) W\left(2 v_{i}\right)}{1-\left|W\left(2 v_{i}\right)\right|^{2}}
$$

(see Eqs. (23) and (25) in Roberts et al. 1987). The $c_{i}$ were used in Eq. (A.3) for the calculation of the aliasing powers rather than the power $\left|D_{i}\left(v_{i}\right)\right|$ of the peak since the peak itself is affected by aliasing of the corresponding negative peak located at $-v_{i}$. The $A_{i}$ are subtracted from the spectrum $D_{i}$ and the resulting spectrum $D_{i+1}=D_{i}-A_{i}$ is the input in the next iteration step.

The factor $g$ in Eq. (A.4) is called the gain factor and takes care of the possibility that the highest peak itself could be an artifact. Therefore only a fraction $(g)$ of the power is used to calculate the alias powers. The iteration process stops after the $N$ th iteration if the power of the highest peak in the subtracted spectrum $D_{N+1}$ is below a given level $P_{\min }$ or if a maximum number $N_{\max }$ of iteration steps is exceeded. The last output $D_{N+1}$ is called the residual spectrum. We used the values $g=0.01, P_{\min }=0.001$ and $N_{\max }=300$. Normally the peak power in the residual spectrum falls below this limit of $P_{\min }=0.001$ before the maximal iteration of $N_{\max }=300$ is reached.

During the iteration process any artifact should be removed and the main output is the clean component spectrum which is defined as

$C(v)=\sum_{i=1}^{N} \int_{-\infty}^{+\infty} c_{i} \delta\left(\hat{v}-v_{i}\right) \mathrm{d} \hat{v}$,

where $\delta(x)$ is the Dirac delta function and the $c_{i}$ are the clean components from Eq. (A.4). The resolution of the clean component spectrum in Eq. (A.5) is given by the artificial frequency spacing $\Delta v$ used in the algorithm (see above). Since the resolution of the spectrum $F$ that results from a discrete data set is $\Delta v \simeq 1 / T$ we have to smooth the clean component spectrum with a beam function $B(v)$. This is necessary because the power of the peaks in the spectra $D_{I}$ is distributed into $n_{B}=\Delta v / T$ frequency bins (points per beam) in the interval $\left[v_{i}-1 / T, v_{i}+1 / T\right]$. The function $B(v)$ is found by a fit of the main peak of the window function at $v=0$.

The final step of the CLEAN algorithm is the calculation of the clean spectrum $S(v)$ that contains the periodogram of the signal where the window function $W(v)$ has been removed. $S(v)$ represents the spectrum $F(v)$ in Eq. (1). $S(v)$ is obtained by adding the residual spectrum $D_{N+1}(v)$ and the smoothed clean component spectrum $C(v)$ :

$S(v)=D_{N+1}(v)+C(v)$.

\section{References}

Appenzeller, I., \& Mundt, R. 1989, A\&AR, 1, 291

Bailer-Jones, C. A. L., \& Mundt, R. 2001, A\&A, 367, 218; 2001, A\&A, 374, 1071 (erratum)

Bessel, M. S. 1983, PASP, 95, 480

Bodenheimer, P. 1995, ARA\&A, 33, 199

Bouvier, J., \& Bertout, C. 1989, A\&A, 211, 99

Bouvier, J., Forestini, \& Allain, M. S. 1997, A\&A, 326, 1023

Camenzind, M. 1990, RvMA, 3, 234

Deeming, T. J. 1975, Ap\&SS, 36, 137

Feigelson, E. D., \& Montmerle, T. 1999, ARA\&A, 37, 363

Flaccomio, E., Micela, G., Sciortino, S., et al. 1999, A\&A, 345, 521

Flaccomio, E., Micela, G., Sciortino, S., et al. 2000, A\&A, 355, 651

Herbig, G. 1954, ApJ, 119, 483

Herbst, W., Herbst, D. K., Grossman, E. J., \& Weinstein, D. 1994, AJ, 108, 1906

Herbst, W., Bailer-Jones, C. A. L., \& Mundt, R. 2001, ApJ, 554, L197

Herbst, W., Bailer-Jones, C. A. L., Mundt, R., Meisenheimer, K., \& Wackermann, R. 2002, A\&A, 396, 513

Horne, J. H., \& Baliunas, S. L. 1986, ApJ, 302, 757

Johnson, H. L. 1966, ARA\&A, 4, 193

Kearns, K. E., \& Herbst, W. 1998, AJ, 116, 261

Kirkpatrick, J. D., \& McCarthy, A. W. Jr. 1994, AJ, 107, 333

Koch, A. 2002, private communication

Königl, A. 1991, ApJ, 370, L39

Kuhi, L. V. 1978, M\&P, 19, 199

Kibng, J. R. 1998, AJ, 116, 254

Lamm, M. H., Bailer-Jones, C. A. L., Mundt, R., \& Herbst, W. 2003, in press (Paper II)

Leggett, S. K. 1992, ApJS, 82, 351 
Mathieu, R. D. 1986, IAU Highlts. Astr., 7, 481

Mathieu, R. D. 2003, in Stellar Rotation, ed. A. Maeder, \& P. Eenens, Proc. IAU Symp., 215

Mahdavi, A., \& Kenyon, J. S. 1998, AJ, 497, 342

Marcy, G. W. 1980, AJ, 85, 230

Monet, D., Bird, A., Canzian, B., et al. 1998, The USNO-A2.0 Catalogue

Najita, J., Edwards, S., Basri, G., \& Carr, J. 2000, in Protostars and Planets IV, ed. V. Mannings, A. P. Boss, \& S. S. Russell (Tucson: Univ. of Arizona Press), 457

Nandy, K., \& Pratt, N. 1972, Ap\&SS, 19, 219

Neri, L. J., Chavarría, C., \& de Lara, E. 1993, A\&AS, 102, 201

Ogura, K. 1984, PASJ, 36, 139

Park, B., Sung, H., Bessel, M., \& Kang, Y. 2000, AJ, 120, 894

Press, W. H., Teulolsky, S. H., Vetterling, W. T., \& Flannery, B. P. 1992, Numerical Recipies in C (Cambridge University Press)

Rebull, L. M., Makidon, R. B., Strom, S. E., et al. 2002, AJ, 123, 1528
Roberts, D. H., Lehar, J., \& Dreher, J. W. 1987, AJ, 93, 968

Rydgren, A. E. 1979, ApJ, 84, 90

Rydgren, A. E., \& Vrba, F. J. 1983, ApJ, 267, 191

Sagar, R., Myakutin, V. I., Piskunov, A. E., \& Dluzhnevskaya, O. B. 1988, MNRAS, 234, 831

Scargle, J. D. 1982, ApJ, 263, 835

Shu, F. H., Najita, J., Ostriker, E., et al. 1994, ApJ, 429, 781

Sung, H., Bessel, M. S., \& Lee, S.-W. 1997, AJ, 114, 2644

Soderblom, D. R., King, J. R., Siess, L., Jones, B. F., \& Fischer, D. 1999, ApJ, 118, 1301

Stassun, K. G., Mathieu, R. D., Mazeh, T., \& Vrba, F. J. 1999, AJ, 117, 2941

Vasilevskis, S., Sanders, W. L., \& Balz, A. G. A. 1965, 70, 797

Vogel, S. N., \& Kuhi, L. V. 1981, AJ, 245, 960

Vrba, F. J., Rydgren, A. E., Chugainov, P. F., Shakovskaya, N. I., \& Bruce Weaver, W. 1989, AJ, 97, 483

Walker, M. F. 1956, ApJS, 2, 365 